\title{
PATRONES MODELO DE MORTALIDAD PARA MÉXICO*
}

\author{
Virgillo Partida $B$. \\ El Colegio de México
}

\section{INTRODUCCIÓN}

EN MUCHOS DE LOS países llamados subdesarrollados es difícil, si no imposible, determinar el nivel y el comportamiento por edad de la mortalidad, debido a la falta de información o, si la hay, a que es incompleta o adolece de problemas de cobertura y mala declaración de edad. Para poder conocer el nivel y el comportamiento por edad del fenómeno en estos países, en los últimos veinticinco años-se han ideado patrones modelo de mortalidad por edad -mejor conocidos como "tablas modelo de mortalidad"-, diseñados para proporcionar un comportamiento completo y adecuado del fenómeno en base a una escasa información disponible.

Los patrones modelo de mortalidad construidos hasta ahora son los de las Naciones Unidas (1963), los de Gabriel y Ronen (1958), los de Coale y Demeny (1966), los de Ledermann (1969), el sistema logito de Brass (1974) y su corrección por Zaba (1979), y los de Bourgeois-Pichat (1970) que están basados en el análisis factorial de la mortalidad por edad hecho por Ledermann y Breas (1959).

México es uno de los pocos países en desarrollo que con menos frecuencia ha recurrido al uso de estos modelos de mortalidad, ya que cuenta con información continua sobre defunciones desde 1922; aunque, si bien la calidad de los datos ha ido mejorando al correr el período post-revolucionario, no deja aún de presentar problemas de cobertura y mala declaración de edad. ${ }^{2}$

Cualquiera de los modelos construidos hasta ahora, presenta una sensible diferencia con el comportamiento de la mortalidad por edad observado en México a partir de los 20 años, como se puede observar dentro del rectángulo en la gráfica 1 : las probabilidades de fallecer en el modelo

* El autor agradece a los profesores Carmen A. Miró y Manuel Ordorica, sus valiosos consejos y sugerencias para la realización de la investigación.

1 Véanse, entre otros, Arriaga (1968), Ordorica et al. (1975), Cordero (1968). 
Gráfica 1

COMPARACIÓN ENTRE EL Tí́lico COMPORTAMIENTO POR EDAD DE LA MORTALIDAD OBSERVADO EN MÉXICO Y EN CUALQUIERA DE LOS MODELOS CONSTRUIDOS HASTA AHORA

(Probabilidades de fallecer por mil)

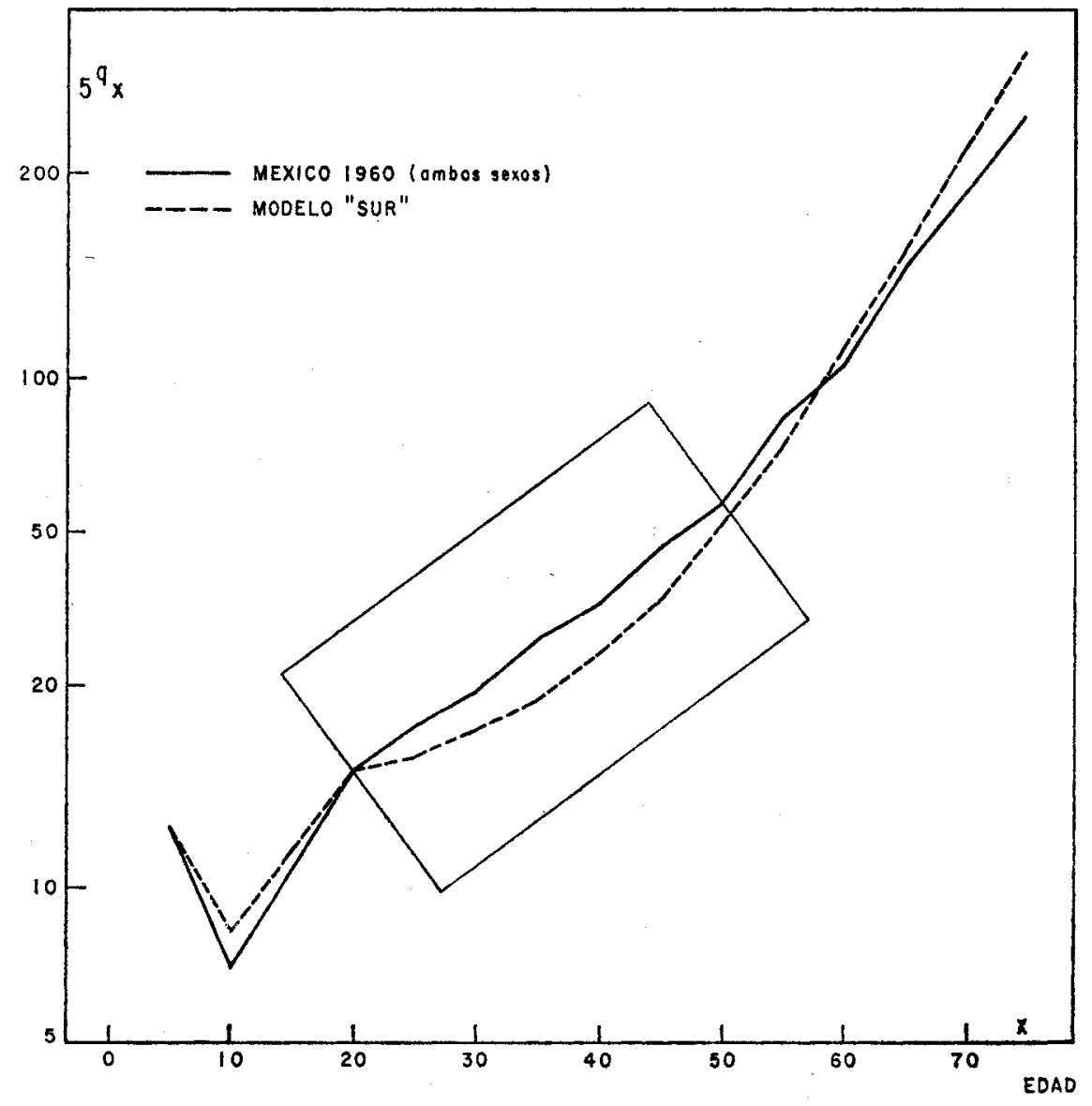

Fuentes: Para México, alrededor de 1960 , se obtuvieron de las tasas centrales de mortalidad del trabajo de Benítez y Cabrera (1967).

Para el modelo "Sur", se obtuvieron, suponiendo un índice de masculinidad al nacimiento de 105, en el nivel 16 del trabajo de Coale y Demeny (1966). 
"Sur" de Coale y Demeny (1966) ${ }^{2}$ siguen la forma de una curva cóncava vista desde arriba, mientras que, el típico patrón mexicano sigue la forma de una curva convexa. A raíz de esta diferencia, surgió la idea de construir patrones modelo de mortalidad propios para México.

\section{INFORMACIÓN DISPONIBLE Y UTILIZADA}

Para la construcción de los modelos se dispuso de 75 patrones observados en México, uno para cada sexo por separado:

i) 64 a nivel estatal, para cada entidad federativa, para los trienios 1959-1961 y 1972-1974;

ii) Ocho a nivel regional en 1970; $\mathrm{y}$

iii) Tres a nivel nacional, para alrededor de 1950,1960 y $1970 .^{3}$

Todos estos patrones son de momento y su representación inicial está dada por tasas centrales por edad, referidas a períodos cortos de tiempo. A partir de un análisis del efecto que pudieran tener el subregistro, la mala declaración de edad y la migración interna, en el caso de los patrones a nivel estatal y regional sobre el nivel y el comportamiento por edad de las tasas centrales de mortalidad, se extrajeron las siguientes conclusiones: ${ }^{4}$

a) El subregistro de las defunciones en las estadísticas vitales y en las poblaciones medias estimadas, después del primer año de vida, puede ser considerado como despreciable y compensado en las tasas centrales de mortalidad; por lo tanto, los patrones modelo que se obtengan representarán de forma adecuada el nivel del fenómeno, a partir de un año de edad.

b) Los patrones modelo serán aplicables, de manera muy aproximada, a la población residente en las diversas entidades federativas de la República Mexicana.

c) Las irregularidades observadas en el comportamiento por edad se deben fundamentalmente a la mala declaración de la misma en las defunciones de las estadísticas vitales.

d) En base a los datos de la Encuesta Mexicana de Fecundidad, se prueba que existe cierto grado de subestimación, a nivel nacional, del valor de la probabilidad de fallecer en el primer año de vida $\left({ }_{1} q_{0}\right)$ obtenida de las estadísticas vitales.

2 Se tomó la esperanza de vida a los 5 años de edad, en la obtención del nivel correspondiente en la familia "Sur" de Coale y Demeny (1966), con el fin de que no interfírieran los problemas de registro antes de los 5 años de edad. La elección del modelo "Sur" responde a que diferentes autores han considerado este modelo como el más apropiado al caso mexicano.

3 Los patrones estatales alrededor de 1960 fueron estimados en el Centro de Estudios Económicos y Demográficos de El Colegio de México y están inéditos; los estatales alrededor de 1973 fueron obtenidos por los alumnos de la Maestría en Demografía 1977-1979 del mismo Centro y también están inéditos. Los patrones regionales fueron tomados de Ordorica y otros (1976), y los nacionales de Benítez y Cabrera (1967) y de Cabrera, Ordorica y García (1973).

4 Para un análisis más detallado, véase Partida (1980), pp. 51-69. 
Antes de construir los modelos y a fin de que estos no configuraran patrones modelo mal comportados, se consideró necesario corregir tanto el comportamiento irregular por edades como la subestimación durante el primer año de vida, en los 75 patrones considerados.

\section{El patrón estándAR MEXICANo}

Para corregir estas irregularidades en los patrones considerados se eligió el método logito de Brass. ${ }^{5}$ Sea $1(x)$ la probabilidad de sobrevivir desde el nacimiento hasta la edad exacta $x$ en un patrón de mortalidad, su logito, $Y(x)$, se define como:

$$
Y(x)=\frac{1}{2} \ln \left[\frac{1-1(x)}{1(x)}\right] ;
$$

la ecuación fundamental del sistema logito establece que dos patrones de mortalidad pueden ser relacionados linealmente a través de sus logitos, es decir,

$$
Y_{o}(x)=\alpha+\beta \cdot Y_{s}(x)
$$

donde, $Y_{o}(x)$ son los logitos de un patrón observado a corregir, y $Y_{s}(x)$ los de un patrón bien comportado o estándar, con el cual, y habiendo determinado los parámetros $\alpha$ y $\beta$, se obtienen valores ajustados para $Y_{o}(x)$ y la función $1(x)$ de la tabla de mortalidad aplicando inversamente la función logito.

Si se desean obtener patrones corregidos congruentes con los patrones observados, al utilizar el método logito es necesario que el patrón estándar represente satisfactoriamente el comportamiento por edad de la mortalidad en estudio; de donde, las diferentes curvaturas que se presentan entre el típico comportamiento observado en México y en cualquiera de los modelos construidos hasta ahora, entre los 20 y 50 años de edad (citadas anteriormente) hacen necesario obtener un patrón estándar particular del comportamiento por edad de la mortalidad mexicana.

¿Cómo obtener un estándar propio para la mortalidad mexicana? Brass (1974, p. 144) sugiere que "la tabla estándar debe ser algún tipo de promedio. Si dos tablas de mortalidad han de tener cada cual una relación en línea recta con una tercera en la escala logito, entonces también deben ser lineales en su relación entre sî".

5 Para una explicación más detallada del sistema logito, véase Brass (1973 y 1974), la corrección al ajuste en las edades extremas propuesta por Zaba (1979) y el sistema de tablas modelo de vida, a partir del método logito, construido por Carrier y Hobcraft (1975). 
Se obtuvieron dos estándares mexicanos: uno para hombres y otro para mujeres, con el fin de que al corregir los 75 patrones considerados y en la eventual aplicación del sistema logito se preservara un adecuado diferencial por sexo. En ambos estándares se siguió el procedimiento que se describe a continuación.

Debido a la presencia de cierto grado de subestimación en la mortalidad en el primer año de vida, se evitó hacer cualquier tipo de promedio sobre medida alguna del fenómeno en este intervalo de edades. La probabilidad de fallecer entre 1 y 5 años de edad exacta $\left({ }_{4} q_{1}\right)$ para el estándar mexicano fue obtenida como el promedio aritmético de las correspondientes probabilidades en los 75 patrones considerados.

La estimación de la probabilidad de fallecer en el primer año de edad $\left({ }_{1} q_{0}\right)$ fue hecha a partir del valor de ${ }_{4} q_{1}$ mediante las fórmulas obtenidas a partir de las historias de embarazos de la Encuesta Mexicana de Fecundidad:

$$
\begin{aligned}
& \text { Para hombres }{ }_{1} q_{0}=2.29797 \cdot{ }_{4} q_{1}, \mathrm{y} \\
& \text { Para mujeres } \quad{ }_{1} q_{0}=1.81374{ }^{\circ}{ }_{4} q_{1}{ }^{\circ}
\end{aligned}
$$

Si bien estas relaciones tienen la objeción de ser rígidas, ya que no distinguen niveles en ${ }_{1} q_{1}$, sino que suponen que la razón ${ }_{1} q_{0} /{ }_{1} q_{1}$ es constante en todas las entidades federativas, quizás más que cuestionables que a nivel nacional o regional, tienen dos ventajas: están referidas exclusivamente a la mortalidad mexicana, cuando en los modelos construidos hasta ahora las estimaciones están basadas, principalmente, en la experiencia de países desarrollados; y, por otro lado, están referidas a un período, 19591974, dentro del cual se encuentran los patrones a corregir.

Con los valores promedio obtenidos para ${ }_{4} q_{1}$ y las relaciones (2) se obtuvieron las ${ }_{1} q_{0}$ correspondientes a los estándares mexicanos.

El grupo de edades 5-9 años es considerado universalmente, el que presenta menos problemas de registro y mala declaración de edad, tanto en las defunciones de las estadísticas vitales como en las poblaciones censadas; así, el cociente de mortalidad ${ }_{5} q_{5}$ fue obtenido a partir del promedio aritmético de las tasas centrales ${ }_{5} M_{5}$ en los 75 patrones y de la conocida fórmula de Reed y Merrell (1939), resultando congruente en ambos patrones estándar, su valor con el comportamiento por edad del fenómeno.

Para calcular en los estándares las probabilidades de fallecer a partir de los diez años, se buscó un método que permitiera obtenerlos a partir de los promedios aritméticos de las respectivas tasas centrales en los patrones considerados y que, de existir irregularidades en el comportamiento por edad de estas tasas promedio, el ajuste que fuera requerido fuera el mínimo posible y estuviera lo más cercano al comportamiento de dichas tasas.

6 Para una descripción de la forma como fueron determinados estos factores, véase Partida (1980), pp. 81-83. 
El método elegido fue el desarrollado por Arriaga (1968), donde el posible ajuste se hace en el comportamiento de las tasas instantáneas de mortalidad que es más regular que en las tasas centrales o en las probabilidades de fallecer. El método parte del supuesto de que se da la ley de Gompertz en cada intervalo quinquenal de edades:

$$
u_{\alpha}=B \cdot c^{\alpha}, \text { para } X \leq \alpha \leq X+S
$$

y, dado que, $u_{\alpha}=-D_{\alpha}\left(\ln 1_{\alpha}\right)$, donde $D_{\alpha}$ significa la primera derivada con respecto a $\alpha$, integrando entre $x$ y $x+u$ y sustituyendo valores se tiene finalmente que:

$$
\ln \left({ }_{5} p_{x}\right)=-5\left[\frac{u_{x+5}-u_{x}}{1 n\left(u_{x+5} / u_{x}\right)}\right] \cdot{ }^{7}
$$

Para poder aplicar esta fórmula es necesario obtener las tasas instantáneas de mortalidad para las diferentes edades que se consideren, las cuales, dado que están referidas al preciso instante de la edad exacta $x$, no se pueden obtener de las defunciones de las estadísticas vitales, sino que debe recurrirse a alguna fórmula de aproximación.

Las fórmulas empleadas fueron las desarrolladas por Barral Souto y Somoza (1953) que suponen el comportamiento de un polinomio de cuarto grado, con respecto a la edad, en las tasas instantáneas de mortalidad:

$$
\begin{aligned}
& \left.u_{10}=\frac{1}{4}{ }_{5} M_{5}+6 \cdot{ }_{5} M_{10}-4 \cdot{ }_{10} M_{10}+{ }_{15} M_{10}\right], \mathrm{y}, \\
& u_{x}=\frac{1}{6}\left[-{ }_{10} M_{x-10}+4 \cdot{ }_{5} M_{x-5}+4 \cdot{ }_{5} M_{x}-{ }_{10} M_{x}\right] \text { para } x=15,20,25, \ldots
\end{aligned}
$$

La última edad considerada para la estimación de las tasas instantáneas de mortalidad fue 65 años, debido a que la tasa central ${ }_{5} M_{75}$ fue menor que la que se esperaba de la tendencia observada en las tasas de los grupos de edades anteriores, en casi todos los 75 patrones considerados, y, de considerar el promedio aritmético de las ${ }_{5} M_{75}$, pudiera tenerse un descenso inaceptable después de los 65 años de edad en los patrones estándar mexicanos.

Los valores de $u_{x}$ calculados de 10 a 65 años de edad, aparecen en el cuadro 1 y en la gráfica 2 , donde se puede observar un patrón bien comportado en el caso masculino, en el cual no se hizo ajuste alguno; pero, en el caso femenino se pueden observar dos pequeñas irregularidades, la primera a los 30 años y la segunda a los 60 . La primera irregularidad

7 El desarrollo de esta fórmula puede verse en Arriaga (1968), p. 6. 


\section{Cuadro 1}

TASAS INSTANTÁNEAS DE MORTALIDAd, PROBABILIDAdES DE FALlecer Y LOGITOS DE LOS PATRONES ESTÁNDAR MEXICANOS

\begin{tabular}{|c|c|c|c|}
\hline$\underset{x}{\operatorname{Edad}}$ & $\begin{array}{l}\text { Tasa instantánea } \\
\text { de mortalidad } u_{x} \\
\text { (Por mil) }\end{array}$ & $\begin{array}{l}\text { Probabilidad de } \\
\text { fallecer } n^{q} \times\end{array}$ & $\begin{array}{l}\text { Logitos } \\
Y(x)\end{array}$ \\
\hline \multicolumn{4}{|c|}{ Hombres } \\
\hline $\begin{array}{l}0 \\
1 \\
5 \\
10 \\
15 \\
20 \\
25 \\
30 \\
35 \\
40 \\
45 \\
50 \\
55 \\
60 \\
65 \\
70 \\
75 \\
80 \\
85\end{array}$ & $\begin{array}{r}1.3122 \\
1.6029 \\
2.9235 \\
4.0060 \\
4.4961 \\
5.4471 \\
6.5909 \\
8.1549 \\
10.4934 \\
13.7095 \\
18.8707 \\
.26 .5739\end{array}$ & $\begin{array}{l}0.084236 \\
0.036657 \\
0.010244 \\
0.007237 \\
0.010927 \\
0.017035 \\
0.021008 \\
0.024478 \\
0.029559 \\
0.036060 \\
0.045316 \\
0.058376 \\
0.077589 \\
0.106416 \\
0.149922 \\
0.223665 \\
0.353349 \\
0.591131 \\
1.000000\end{array}$ & $\begin{array}{r}-1.193068 \\
-1.006691 \\
-0.964586 \\
-0.936646 \\
-0.896795 \\
-0.839461 \\
-0.775496 \\
-0.708479 \\
-0.635957 \\
-0.557083 \\
-0.469225 \\
-0.369613 \\
-0.253946 \\
-0.116272 \\
0.051570 \\
0.270044 \\
0.581629 \\
1.113558\end{array}$ \\
\hline \multicolumn{4}{|c|}{ Mujeres } \\
\hline $\begin{array}{l}0 \\
1 \\
5 \\
10 \\
15 \\
20 \\
25 \\
30 \\
35 \\
40 \\
45 \\
50 \\
55 \\
60 \\
65 \\
70 \\
75 \\
80 \\
85\end{array}$ & $\begin{array}{r}1.1844 \\
1.1734 \\
2.0163 \\
2.8270 \\
3.4928 \\
4.3078 \\
5.3037 \\
6.5183 \\
8.3271 \\
11.1705 \\
16.6776 \\
25.1985\end{array}$ & $\begin{array}{l}0.066483 \\
0.037696 \\
0.009741 \\
0.005877 \\
0.007755 \\
0.011923 \\
0.015618 \\
0.019243 \\
0.023658 \\
0.029021 \\
0.036256 \\
0.047244 \\
0.066396 \\
0.091147 \\
0.131946 \\
0.201425 \\
0.324256 \\
0.550455 \\
1.000000\end{array}$ & $\begin{array}{r}-1.305115 \\
-1.078939 \\
-1.033584 \\
-1.007939 \\
-0.975752 \\
-0.929459 \\
-0.873835 \\
-0.811724 \\
-0.743107 \\
-0.668034 \\
-0.584968 \\
-0.489929 \\
-0.374142 \\
-0.238277 \\
-0.071184 \\
0.145697 \\
0.450146 \\
0.950859\end{array}$ \\
\hline
\end{tabular}

fue corregida estimando los valores de $u_{30}$ y $u_{35}$ a través de la parábola coincidente, en escala logarítmica, con las tasas instantáneas a las edades 25,40 y 45 años. La segunda irregularidad, a los 60 años de edad, fue ajustada simultáneamente con las probabilidades de fallecer a partir de los 70 años de edad.

Después de observar el comportamiento por edad de las tasas centrales promedio, se decidió obtener las probabilidades ${ }_{5} q_{65}$ y ${ }_{5} q_{70}$, en ambos estándares, a través de las tasas centrales promedio ${ }_{5} M_{65}$ y ${ }_{5} M_{70}$ y de la fórmula de Reed y Merrell (1939). Finalmente, del comportamiento por edad en las probabilidades de fallecer en ambos estándares (véase gráfica 3 ), se decidió estimar las probabilidades de fallecer ${ }_{5} q_{75}$ y ${ }_{5} q_{80}$, en el caso masculino, mediante la parábola coincidente, en escala logarítmica, con 


\section{Gráfica 2}

Patrones estándar meXicanos:

TASAS INSTANTÁNEAS DE MORTALIDAD

(Por mil)

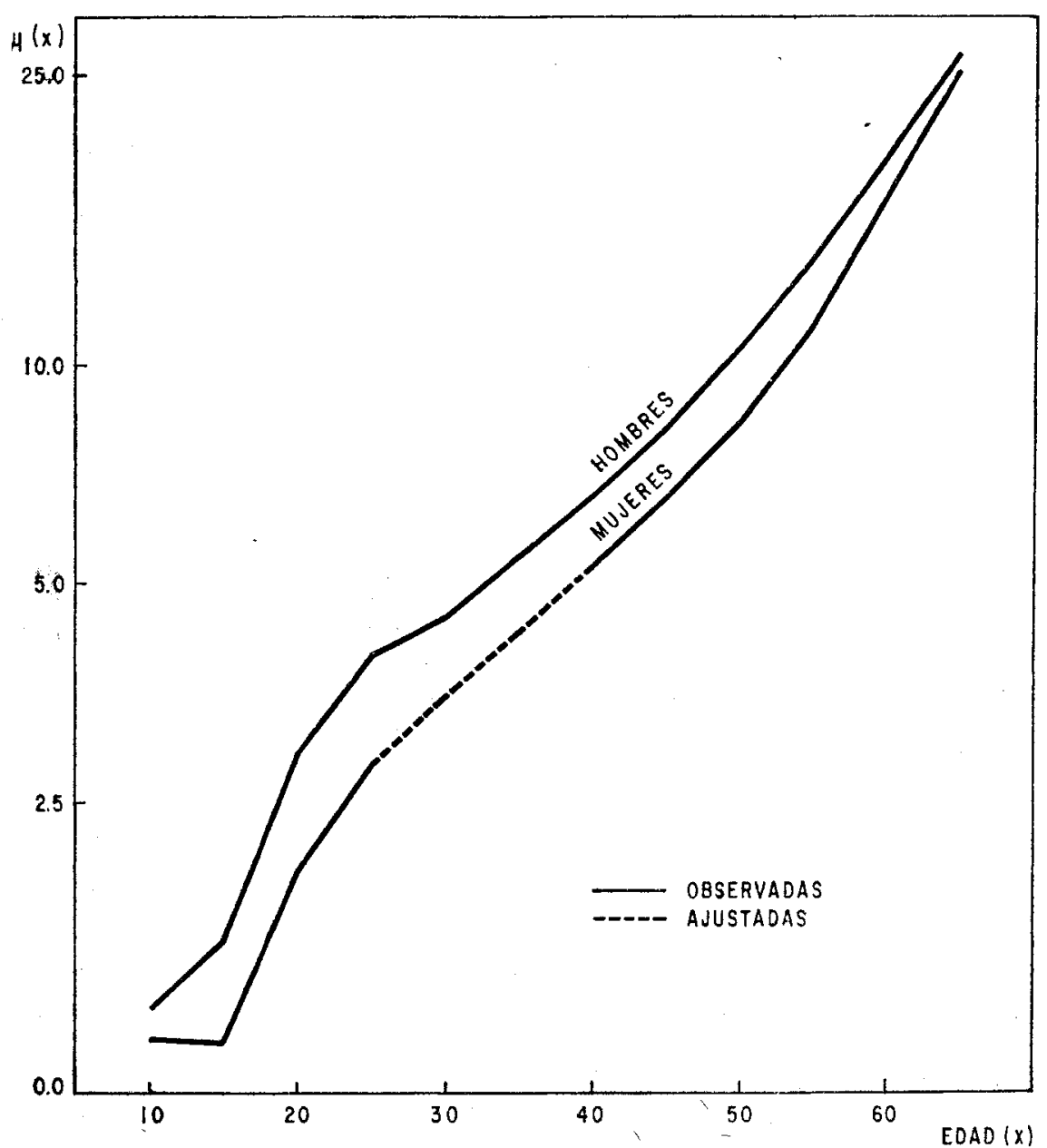

Fuente: Cuadro 1.

los cocientes ${ }_{5} q_{60},{ }_{5} q_{65}$ y ${ }_{5} q_{70} ; \mathrm{y}$, los cocientes ${ }_{5} \mathrm{q}_{60},{ }_{5} q_{75} y_{5} q_{80}$, en el caso femenino, de la parábola coincidente, en escala logarítmica, con las probabilidades de fallecer ${ }_{5} q_{55},{ }_{5} q_{65}$ y ${ }_{5} q_{70}$. Estos últimos ajustes permitieron asignar el valor de 1 a la probabilidad de fallecer a los 85 años de edad, tanto en hombres como en mujeres, y considerar en el último intervalo de edades, abierto, una edad inicial de 85 años. 
Los valores de las probabilidades de fallecer y de los logitos de los patrones estándar, aparecen en el cuadro 1.

La corrección de las irregularidades observadas en los 75 patrones se hizo, como se expuso antes, mediante la aplicación del sistema logito con los estándares mexicanos obtenidos; sin embargo, antes de aplicar este método se consideró necesario corregir la probabilidad de fallecer en el primer año de vida para los casos en que hubiera estado subestimada, pues

\section{Gráfica 3}

\section{Patrones estándar mexicanos: \\ PROBABILIDADES DE FALLECER \\ (Por mil)}

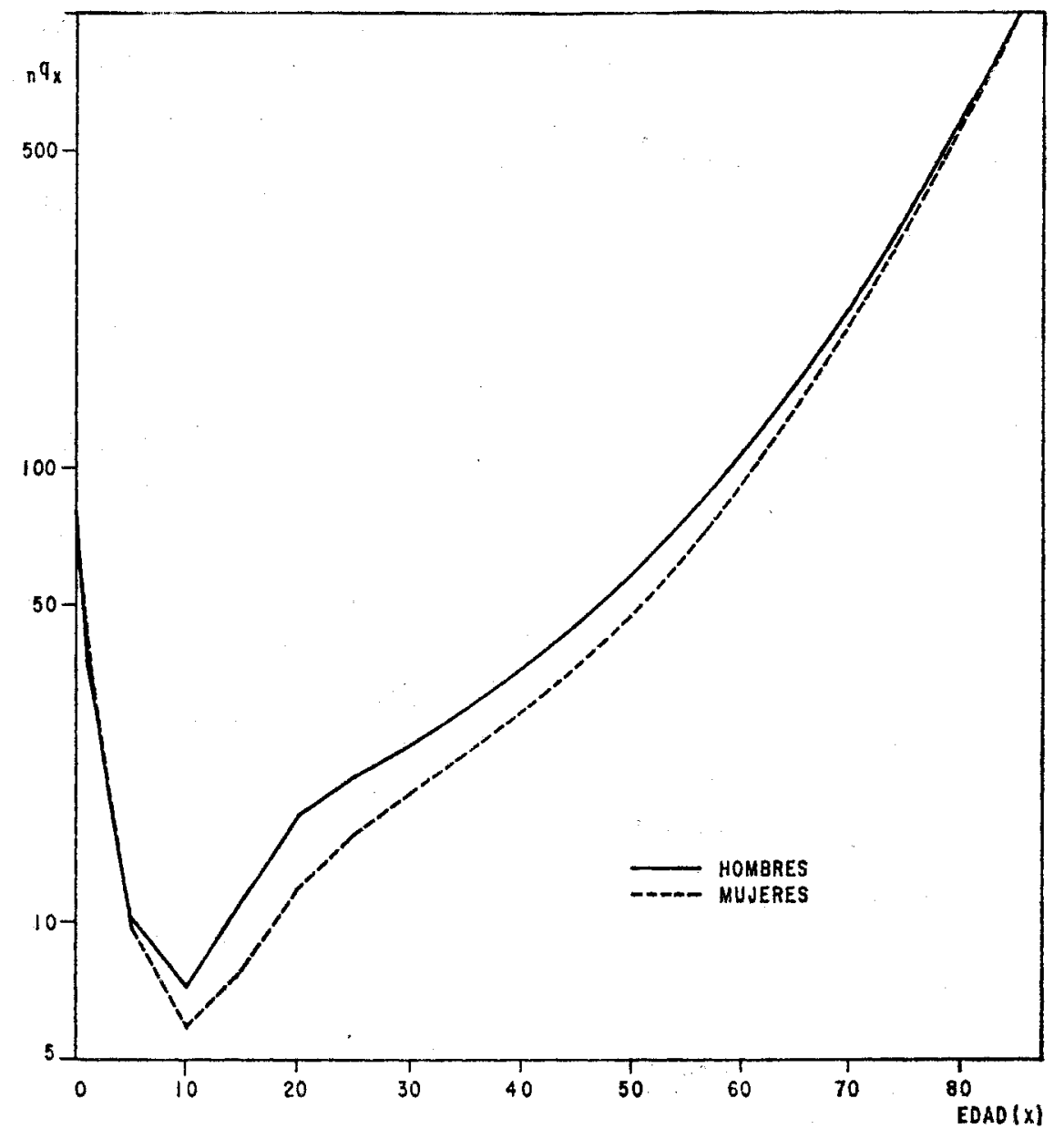

FuENTE: Cuadro 1. 
de haber sido este el caso, se corría el riesgo de tener subestimado el nivel del patrón corregido correspondiente. ${ }^{8}$

La corrección de la posible subestimación en el cociente ${ }_{1} q_{0}$ fue hecha aplicando los factores que aparecen en las relaciones (2) al valor observado de ${ }_{4} q_{1}$, donde, si el valor observado de ${ }_{1} q_{0}$ fue mayor al estimado se dejó el valor observado, suponiendo que en tal caso la subestimación era inexistente o mínima. ${ }^{9}$

Finalmente, al analizar la forma en que el subregistro de defunciones en el primer año de vida y la migración interna afectan la estimación de ${ }_{4} q_{1}$, se concluyó que el posible grado de sobre o subestimación de su valor debía ser a lo sumo de un $10 \%$ en los 75 patrones, con lo cual, para la construcción de los modelos se tomaron como criterios para determinar los patrones definitivos los siguientes:

a) Si la probabilidad ${ }_{4} q_{1}$ en el patrón corregido por el método logito no se alejó más de un $10 \%$, hacia arriba o hacia abajo del valor observado, se tomó íntegro el patrón corregido; y,

b) Si la probabilidad ${ }_{4} q_{1}$ en el patrón corregido se alejó más de un $10 \%$ del valor observado, se asignó el valor obervado y su correspondiente ${ }_{1} q_{0}$ al patrón corregido por el método logito, y el patrón resultante fue tomado como definitivo. ${ }^{10}$

\section{Patrones modelo obtenidos directamente de dos entradas}

Disponer de patrones estándar propios para México representa un primer tipo de metodología para la obtención de patrones modelo de mortalidad, no obstante estos estándares se concibieron, en principio, únicamente para la corrección de los patrones considerados. La idea inicial fue construir patrones modelo similares a los de Ledermann (1969): determinar, hasta donde fuera posible, el nivel global y el comportamiento por edad del fenómeno a través de ecuaciones que relacionaran las probabilidades de fallecer entre dos edades exactas $\left({ }_{n} q_{x}\right)$ con dos medidas de la mortalidad o entradas pensando que lo más indicado era considerar una entrada referida a la mortalidad en edades jóvenes y la otra en edades adultas. Estas entradas, de acuerdo a los estudios de Bourgeois-Pichat (1962 y 1970), fueron elegidas un tanto arbitrariamente: las probabilidades de fallecer entre los 5 y 15 y entre los 45 y 65 años de edad $\left({ }_{10} q_{55}\right.$ y $\left.{ }_{20} y_{45}\right)$.

Las razones por las que no se consideró la mortalidad antes de los 5 años de edad, son: la subestimación en la mortalidad infantil y el largo

8 La demostración matemática puede verse en Partida (1980), pp. 85-86.

${ }^{\theta}$ La subestimación en la probabilidad de fallecer en el primer año de vida en los 75 patrones puede verse en ibid., "Anexo II", pp. 130-169.

10 El análisis del efecto que el subregistro de defunciones en el primer año de vida y la migración interna puedan tener en el valor de el cociente ${ }_{4} q_{1}$ se presenta en ibid., pp. 57 y 62-65. 
proceso en la construcción y corrección - por subregistro y migración interna - en los diagramas de Lexis para determinar la medida correspondiente a los primeros cinco años de vida, dado que complicarían bastante la aplicación de los modelos.

Las ecuaciones que relacionan las probabilidades de fallecer con las dos entradas fueron obtenidas mediante regresiones lineales dobles:

i) tomando las probabilidades y las entradas multiplicadas por mil,

$$
{ }_{n} Q_{x}=a_{0 x}+a_{1 x} \cdot{ }_{10} Q_{5}+a_{2 x} \cdot{ }_{20} Q_{45} ; \mathrm{y},
$$

ii) tomando los logaritmos decimales de las probabilidades y de las entradas multiplicadas por mil,

$$
{ }_{n} Z_{x}=a_{0 x}^{\prime}+a_{1 x}^{\prime} \cdot{ }_{10} Z_{5}+a_{2 x}^{\prime} \cdot{ }_{20} Z_{45},
$$

donde, ${ }_{n} Q_{x}$ es igual a $1000 \cdot{ }_{n} q_{x} y_{n} Z_{x}$ es el logaritmo decimal de ${ }_{n} Q_{x}$.

Los resultados de las regresiones aparecen en el cuadro 2, donde se podrá observar que fueron estadísticamente satisfactorios, considerando los altos coeficientes de determinación $\left(r^{2}\right)$ en las 72 ecuaciones de regresión. Pero, los coeficientes de las regresiones indican que las probabilidades de fallecer antes de los 10 años de edad están inversamente relacionadas con la mortalidad adulta (los valores negativos de $a_{2 x}$ y $a_{2 x}^{\prime}$ ), y las probabilidades de fallecer después de los 55 años están inversamente relacionadas con la mortalidad en edades jóvenes (los valores negativos de $a_{1 x}$ y $a_{1 x}^{\prime}$ ); lo cual, para cierta pareja de entradas, podría proporcionar valores inaceptables en los cocientes de mortalidad antes de los $10 \mathrm{y}$ después de los 60 años de edad, es decir, patrones modelo demográficamente incoherentes.

Estas relaciones inversas se deben a la gran variación que existe en el número de veces que ${ }_{20} q_{45}$ es mayor que ${ }_{10} q_{\overline{5}}$ en los 75 patrones considerados y, más aún, en el número de veces que una medida de la mortalidad después de cierta edad es respecto a una medida anterior a dicha edad; o, en otros términos, a la gran variación observada en los valores del parámetro $\beta$ del sistema logito en los 75 patrones.

La construcción de modelos intentó controlar la variación del parámetro $\beta$ del sistema logito (ver siguiente inciso); metodológicamente se logró que las entradas quedaran referidas a medidas de mortalidad entre 0 y 35 y entre 35 y 75 años de edad. Así, otra alternativa para relacionar probabilidades de fallecer y entradas fue obtener ecuaciones de regresión análogas a (4) y (5):

$$
\begin{aligned}
& { }_{n} Q_{x}=a_{0 x}+a_{1 x}^{\prime} \cdot{ }_{30} Q_{5}+a_{2 x_{x}} \cdot{ }_{40} Q_{35}, y ; \\
& { }_{n} Z_{x}=a_{0 x}^{\prime}+a_{1 x}^{\prime} \cdot{ }_{30} Z_{5}+a_{2 x}^{\prime} \bullet_{40} Z_{35},
\end{aligned}
$$


donde se consideró la primera entrada a partir de los 5 años de edad para determinar alguna medida del fenómeno desde el inicio de la vida.

Los resultados de los modelos (6) y (7) aparecen en el cuadro 3 , y son estadísticamente satisfactorios, aunque en este caso se dan 3 relaciones inversas entre los cocientes de mortalidad antes de los 25 años de edad y la. segunda entrada y entre las probabilidades de fallecer después de los 65 años de edad y la primera entrada.

Se pudieron haber elegido diferentes parejas de entradas buscando disminuir al maximo o eliminar las relaciones inversas pero hubiera implicado caer en un problema teórico: dejar de lado el análisis factorial de la

Cuadro 2

COEFICIENTES DE REgRESIÓN PARA ESTIMAR LAS PROBABILIDADES DE FALLECER A PARTIR DE LAS ENTRADAS: ${ }_{10} q_{5} \mathrm{Y}_{20} q_{45}$

\begin{tabular}{|c|c|c|c|c|c|c|c|c|}
\hline \multirow[b]{2}{*}{$\underset{\mathbf{x}}{\text { Edad }}$} & \multicolumn{4}{|c|}{ Sin transformacion } & \multicolumn{4}{|c|}{ Con transformacion logarftmica } \\
\hline & $a_{0 x}$ & $a_{1 x}$ & $a_{2 x}$ & $x^{2}$ & $a^{\prime}$ & $a^{\prime}$ & $a^{\prime}$ & $r^{2}$ \\
\hline \multicolumn{9}{|c|}{ HOmbres } \\
\hline $\begin{array}{l}0 \\
1 \\
5 \\
10 \\
15 \\
20 \\
25 \\
30 \\
35 \\
40 \\
45 \\
50 \\
55 \\
60 \\
65 \\
70 \\
75 \\
80\end{array}$ & $\begin{array}{r}50.155 \\
15.789 \\
-\quad 0.027 \\
=\quad 0.116 \\
=\quad 0.321 \\
=\quad 1.793 \\
=\quad 2.424 \\
=\quad 3.1112 \\
=\quad 4.290 \\
=\quad 5.771 \\
-\quad 7.485 \\
=\quad 9.120 \\
-\quad 9.492 \\
-\quad 5.137 \\
14.306 \\
80.360 \\
285.395\end{array}$ & $\begin{array}{r}9.409 \\
4.428 \\
0.606 \\
0.405 \\
0.574 \\
0.811 \\
0.878 \\
0.874 \\
0.866 \\
0.814 \\
0.700 \\
0.459 \\
-0.014 \\
=0.908 \\
-2.511 \\
-5.344 \\
=9.884 \\
-14.703\end{array}$ & $\begin{array}{r}-0.480 \\
-0.221 \\
-0.001 \\
0.001 \\
0.005 \\
0.014 \\
0.027 \\
0.044 \\
0.068 \\
0.101 \\
0.150 \\
0.224 \\
0.337 \\
0.512 \\
0.774 \\
1.180 \\
1.738 \\
2.185\end{array}$ & $\begin{array}{l}0.955 \\
0.988 \\
1.000 \\
1.000 \\
1.000 \\
1.000 \\
1.000 \\
1.000 \\
1.000 \\
1.000 \\
1.000 \\
1.000 \\
1.000 \\
1.000 \\
1.000 \\
0.999 \\
0.999 \\
0.999\end{array}$ & $\begin{array}{r}2.744 \\
2.442 \\
-0.192 \\
-0.447 \\
-0.364 \\
-0.304 \\
-0.376 \\
-0.479 \\
-0.566 \\
-0.652 \\
-0.722 \\
-0.778 \\
-0.806 \\
-0.789 \\
-0.700 \\
-0.468 \\
0.013 \\
0.925\end{array}$ & $\begin{array}{r}1.799 \\
2.200 \\
1.027 \\
0.973 \\
0.917 \\
0.837 \\
0.739 \\
0.634 \\
0.524 \\
0.406 \\
0.279 \\
0.142 \\
-0.006 \\
-0.163 \\
-0.321 \\
-0.465 \\
-0.555 \\
-0.504\end{array}$ & $\begin{array}{r}-1.258 \\
-1.511 \\
-0.030 \\
0.041 \\
0.110 \\
0.206 \\
0.324 \\
0.448 \\
0.575 \\
0.707 \\
0.842 \\
0.981 \\
1.120 \\
1.251 \\
1.357 \\
1.406 \\
1.335 \\
1.022\end{array}$ & $\begin{array}{l}1.000 \\
0.998 \\
1.000 \\
1.000 \\
1.000 \\
1.000 \\
1.000 \\
1.000 \\
1.000 \\
1.000 \\
1.000 \\
1.000 \\
1.000 \\
1.000 \\
1.000 \\
1.000 \\
1.000 \\
1.000\end{array}$ \\
\hline \multicolumn{9}{|c|}{$M \mathfrak{d e r e s}$} \\
\hline $\begin{array}{l}0 \\
1 \\
5 \\
10 \\
15 \\
20 \\
25 \\
30 \\
35 \\
40 \\
45 \\
50 \\
55 \\
60 \\
65 \\
70 \\
75 \\
80\end{array}$ & $\begin{array}{r}41.269 \\
12.407 \\
0.023 \\
=\quad 0.079 \\
=\quad 0.180 \\
=\quad 0.414 \\
=\quad 0.765 \\
=\quad 1.221 \\
=\quad 1.807 \\
=\quad 3.521 \\
=\quad 4.437 \\
=\quad 5.576 \\
=\quad 5.555 \\
-1.838 \\
-14.631 \\
72.742 \\
262.980\end{array}$ & $\begin{array}{r}9.603 \\
5.418 \\
0.644 \\
0.365 \\
0.454 \\
.0 .641 \\
0.747 \\
0.791 \\
0.802 \\
0.763 \\
0.663 \\
0.459 \\
0.020 \\
-0.888 \\
-2.633 \\
=5.963 \\
-11.822 \\
-19.239\end{array}$ & $\begin{array}{r}-0.539 \\
-0.279 \\
-0.001 \\
0.001 \\
0.004 \\
0.011 \\
0.021 \\
0.037 \\
0.059 \\
0.089 \\
0.133 \\
0.202 \\
0.325 \\
0.503 \\
0.797 \\
1.281 \\
2.005 \\
2.713\end{array}$ & $\begin{array}{l}0.997 \\
0.987 \\
1.000 \\
1.000 \\
1.000 \\
1.000 \\
1.000 \\
1.000 \\
1.000 \\
1.000 \\
1.000 \\
1.000 \\
1.000 \\
1.000 \\
1.000 \\
0.999 \\
0.999 \\
0.999\end{array}$ & $\begin{array}{r}2.753 \\
1.954 \\
-0.170 \\
-0.490 \\
-0.448 \\
-0.364 \\
-0.381 \\
-0.438 \\
-0.507 \\
-0.584 \\
-0.656 \\
-0.710 \\
-0.730 \\
-0.751 \\
-0.696 \\
-0.513 \\
-0.083 \\
0.804\end{array}$ & $\begin{array}{r}1.877 \\
2.196 \\
1.025 \\
0.968 \\
0.918 \\
0.849 \\
0.761 \\
0.661 \\
0.550 \\
0.430 \\
0.301 \\
0.159 \\
-0.001 \\
-0.177 \\
-0.361 \\
-0.538 \\
-0.664 \\
-0.634\end{array}$ & $\begin{array}{r}-1.337 \\
-1.297 \\
-0.027 \\
0.045 \\
0.104 \\
0.183 \\
0.285 \\
0.399 \\
0.522 \\
0.654 \\
0.792 \\
0.937 \\
1.089 \\
1.247 \\
1.386 \\
1.476 \\
1.445 \\
1.149\end{array}$ & $\begin{array}{l}1.000 \\
0.997 \\
1.000 \\
1.000 \\
1.000 \\
1.000 \\
1.000 \\
1.000 \\
1.000 \\
1.000 \\
1.000 \\
1.000 \\
1.000 \\
1.000 \\
1.000 \\
1.000 \\
1.000 \\
1.000\end{array}$ \\
\hline
\end{tabular}

${ }^{\mathrm{a}} Q_{n}=a_{0 x}+a_{1 x} \cdot{ }_{10} Q_{5}+a_{2 x} \cdot{ }_{20} Q_{45}$

${ }_{n} Z_{x}=a_{0 x}^{\prime}+a_{1_{x}}^{\prime} \cdot{ }_{10} Z_{5}+a_{2 x}^{\prime} \cdot{ }_{20} Z_{4,5}$

donde, ${ }_{n} Q_{x}$ es igual a $1000 \cdot{ }_{n} q_{x} \mathrm{y}_{n} \mathrm{Z}_{x}$ es igual al logaritmo decimal de ${ }_{n} Q_{x}$ 
mortalidad por edades hecho por Ledermannn y Breas (1959) y su interpretación por Bourgeois-Pichat (1962 y 1970).

Considerando que de las ecuaciones (4) y (6) pudieran obtenerse incluso valores negativos en las probabilidades de fallecer que presentaron relación inversa con alguna de las entradas (algo que no puede ocurrir en las ecuaciones (5) y (7) ya que el contradominio de la función logaritmo son los números reales positivos), y considerando que las entradas ${ }_{30} q_{\text {. }}$ $\mathrm{y}_{40} q_{35}$ comprenden intervalos de edades suficientemente amplios (desde los 5 hasta los 75 años) en los que se puede esperar que el nivel y comportamiento por edad de la mortalidad observada esté mejor representado,

Cuadro 3

COEFICIENTES DÉ REgRESIÓN PARA ESTIMAR LAS PROBABILIDADES DE FALLECER A PARTIR DE LAS ENTRADAS: ${ }_{30} q_{5} \mathrm{Y}_{41} q_{3 \pi}$

\begin{tabular}{|c|c|c|c|c|c|c|c|c|}
\hline \multirow{2}{*}{$\underset{x}{E d a d}$} & \multicolumn{4}{|c|}{ Sin transformacion a/ } & \multicolumn{2}{|c|}{ Con transformacion } & \multicolumn{2}{|c|}{ logarstmica } \\
\hline & $a_{0 x}$ & $a_{I x}$ & ${ }^{a} 2 x$ & $x^{2}$ & $\begin{array}{l}a^{\prime} \\
0 x\end{array}$ & $a^{\prime}$ & $a^{\prime}$ & $x^{2}$ \\
\hline \multicolumn{9}{|c|}{ H $\circ \mathrm{mb} \mathrm{res}$} \\
\hline $\begin{array}{c}0 \\
1 \\
5 \\
10 \\
15 \\
20 \\
25 \\
30 \\
35 \\
40 \\
45 \\
50 \\
55 \\
60 \\
65 \\
70 \\
75 \\
80\end{array}$ & $\begin{array}{r}92.502 \\
36.414 \\
1.020 \\
0.431 \\
0.277 \\
=\quad 0.366 \\
=\quad 3.649 \\
=\quad 5.339 \\
=\quad 8.690 \\
=\quad 13.484 \\
-20.016 \\
=29.337 \\
=42.082 \\
-57.213 \\
-69.408 \\
-49.713 \\
112.586\end{array}$ & $\begin{array}{r}2.076 \\
0.985 \\
0.151 \\
0.102 \\
0.147 \\
0.212 \\
0.237 \\
0.245 \\
0.258 \\
0.264 \\
0.264 \\
0.246 \\
0.191 \\
0.065 \\
-0.194 \\
-0.689 \\
-1.541 \\
-2.539\end{array}$ & $\begin{array}{r}-0.340 \\
-0.160 \\
-0.007 \\
-0.004 \\
-0.004 \\
-0.002 \\
0.004 \\
0.012 \\
0.023 \\
0.040 \\
0.066 \\
0.105 \\
0.167 \\
0.265 \\
0.41 .7 \\
0.658 \\
1.001 \\
1.300\end{array}$ & $\begin{array}{l}0.992 \\
0.987 \\
1.000 \\
1.000 \\
1.000 \\
1.000 \\
1.000 \\
1.000 \\
1.000 \\
1.000 \\
1.000 \\
1.000 \\
0.999 \\
0.999 \\
1.000 \\
1.000 \\
1.000 \\
0.999\end{array}$ & $\begin{array}{r}3.239 \\
3.087 \\
-0.457 \\
-0.753 \\
-0.708 \\
-0.702 \\
-0.840 \\
-1.013 \\
-1.171 \\
-1.328 \\
-1.473 \\
-1.601 \\
-1.700 \\
-1.749 \\
-1.707 \\
-1.484 \\
-0.931 \\
0.210\end{array}$ & $\begin{array}{r}1.967 \\
2.424 \\
1.264 \\
1.211 \\
1.156 \\
1.076 \\
0.979 \\
0.875 \\
0.765 \\
0.646 \\
0.516 \\
0.375 \\
0.219 \\
0.051 \\
-0.124 \\
-0.293 \\
-0.421 \\
-0.423\end{array}$ & $\begin{array}{r}-1.873 \\
-2.294 \\
-0.361 \\
-0.271 \\
-0.182 \\
-0.058 \\
0.095 \\
0.256 \\
0.423 \\
0.597 \\
0.778 \\
0.966 \\
1.158 \\
1.345 \\
1.508 \\
1.611 \\
1.571 \\
1.237\end{array}$ & $\begin{array}{l}1.000 \\
0.998 \\
1.000 \\
1.000 \\
1.000 \\
1.000 \\
1.000 \\
1.000 \\
1.000 \\
1.000 \\
1.000 \\
1.000 \\
1.000 \\
1.000 \\
1.000 \\
1.000 \\
1.000 \\
1.000\end{array}$ \\
\hline \multicolumn{9}{|c|}{$M u j \in r e s$} \\
\hline $\begin{array}{l}0 \\
1 \\
5 \\
10 \\
15 \\
20 \\
25 \\
30 \\
35 \\
40 \\
45 \\
50 \\
55 \\
60 \\
65 \\
70 \\
75 \\
80\end{array}$ & $\begin{array}{r}68.823 \\
26.875 \\
0.670 \\
0.216 \\
0.104 \\
=\quad 0.191 \\
=\quad 0.830 \\
=\quad 1.796 \\
=\quad 5.171 \\
=\quad 7.050 \\
=11.677 \\
=18.059 \\
=26.222 \\
=36.787 \\
=45.160 \\
-26.725 \\
121.762\end{array}$ & $\begin{array}{r}2.289 \\
1.308 \\
0.179 \\
0.103 \\
0.130 \\
0.187 \\
0.224 \\
0.248 \\
0.267 \\
0.278 \\
0.280 \\
0.272 \\
0.235 \\
0.102 \\
-0.189 \\
-0.805 \\
-1.985 \\
-3.623\end{array}$ & $\begin{array}{r}-0.311 \\
-0.164 \\
-0.006 \\
-0.003 \\
-0.002 \\
-0.001 \\
0.002 \\
0.008 \\
0.018 \\
0.031 \\
0.051 \\
0.082 \\
0.140 \\
0.226 \\
0.373 \\
0.622 \\
1.008 \\
1.405\end{array}$ & $\begin{array}{l}0.995 \\
0.985 \\
1.000 \\
1.000 \\
1.000 \\
1.000 \\
1.000 \\
1.000 \\
1.000 \\
1.000 \\
1.000 \\
1.000 \\
1.000 \\
1.000 \\
1.000 \\
1.000 \\
0.999 \\
0.999\end{array}$ & $\begin{array}{r}3.022 \\
2.150 \\
-0.476 \\
-0.826 \\
-0.807 \\
-0.754 \\
-0.809 \\
-0.911 \\
-1.027 \\
-1.152 \\
-1.275 \\
-1.381 \\
-1.454 \\
-1.522 \\
-1.505 \\
-1.326 \\
-0.843 \\
0.237\end{array}$ & $\begin{array}{r}1.936 \\
2.336 \\
1.220 \\
1.167 \\
1.121 \\
1.057 \\
0.975 \\
0.881 \\
0.777 \\
0.664 \\
0.540 \\
0.404 \\
0.247 \\
0.071 \\
-0.117 \\
-0.309 \\
-0.468 \\
-0.498\end{array}$ & $\begin{array}{r}-1.753 \\
-1.823 \\
-0.287 \\
-0.203 \\
-0.133 \\
-0.040 \\
0.080 \\
0.216 \\
0.363 \\
0.520 \\
0.686 \\
0.861 \\
1.050 \\
1.246 \\
1.428 \\
1.561 \\
1.566 \\
1.270\end{array}$ & $\begin{array}{l}1.000 \\
0.997 \\
1.000 \\
1.000 \\
1.000 \\
1.000 \\
1.000 \\
1.000 \\
1.000 \\
1.000 \\
1.000 \\
1.000 \\
1.000 \\
1.000 \\
1.000 \\
1.000 \\
1.000 \\
1.000\end{array}$ \\
\hline
\end{tabular}

${ }^{\mathrm{a}} Q_{n}=a_{0 x}+a_{1 \times} \cdot{ }_{30} Q_{5}+a_{2 x} \cdot{ }_{40} Q_{5 ;}$

b $\underset{Z}{Z}=a_{0_{x}}^{\prime}+a_{12}^{\prime} \cdot{ }_{30} Z_{5}+a_{2 x}^{\prime} \cdot{ }_{40} Z_{35}$

donde; ${ }_{n} Q_{x}$ es igual a $1000 \cdot{ }_{n} q_{x}$ y ${ }_{n} Z_{x}$ es igual al logaritmo decimal de ${ }_{n} Q_{x}$ 
se concluyó que los modelos descritos por la ecuación (7) deberían proporcionar los mejores resultados, y modificarla si al obtener un patrón modelo, las probabilidades de fallecer en los primeros cinco años de vida $\left.{ }_{1} q_{0} y_{4} q_{1}\right)$ resultaran incongruentes con la realidad mexicana, pudiendo

\section{Cuadro 4}

INTERVALOS DE ENTRADAS PARA LA APLICACIÓN DE LOS MODELOS (Por mil)

\begin{tabular}{|c|c|c|c|c|}
\hline \multicolumn{5}{|c|}{ a $\circ \mathrm{mbres}$} \\
\hline \multicolumn{3}{|c|}{${ }_{10} q_{5}$} & \multicolumn{2}{|c|}{$20^{q_{45}}$} \\
\hline Mínimo & & Máximo & Minimo & Maximo \\
\hline 5.044 & & 9.736 & 121.234 & 291.653 \\
\hline 9.769 & & 13.057 & 182.546 & 351.908 \\
\hline 13.061 & & 16.230 & 195.823 & 300.185 \\
\hline 16.437 & & 22.385 & 228.094 & 341.837 \\
\hline 23.472 & & 44.846 & 189.687 & 374.016 \\
\hline \multicolumn{3}{|c|}{$30^{q_{5}}$} & \multicolumn{2}{|c|}{$40^{q_{35}}$} \\
\hline 37.049 & & 65.535 & 288.915 & 630.070 \\
\hline 65.788 & & 73.938 & 450.633 & 653.617 \\
\hline 74.383 & & 86.535 & .403 .049 & 588.328 \\
\hline 87.747 & & 108.113 & 432.427 & 610.990 \\
\hline 108.976 & & 166.351 & 508.904 & 672.319 \\
\hline \multicolumn{5}{|c|}{ Mu $j=I e s$} \\
\hline \multicolumn{3}{|c|}{${ }_{10}{ }^{q_{5}}$} & \multicolumn{2}{|c|}{$20^{q_{35}}$} \\
\hline 4.046 & & 7.625 & 114.974 & 231.130 \\
\hline 7.675 & & 10.474 & 167.273 & 244.616 \\
\hline 10.574 & & 14.254 & 177.859 & 232.747 \\
\hline 14.748 & & 22.084 & 170.315 & 256.979 \\
\hline 22.484 & & 44.162 & 204.582 & 296.163 \\
\hline \multicolumn{3}{|c|}{$30^{q_{5}}$} & \multicolumn{2}{|c|}{$40 q_{35}$} \\
\hline 27.165 & & 40.300 & 289.629 & 539.714 \\
\hline 40.319 & & 50.249 & 401.559 & 507.201 \\
\hline 53.403 & & 61.015 & 348.136 & 549.846 \\
\hline 61.609 & & 87.602 & 462.966 & 610.793 \\
\hline 89.722 & 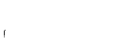 & 154.151 & 459.260 & 607.990 \\
\hline
\end{tabular}

ser ésta la alternativa seguida en la corrección de los 75 patrones considerados, presentada en el inciso anterior.

En el cuadro 4 se presentan intervalos de entradas, obtenidos después de ordenar de menor a mayor el valor de la primera entrada en los 75 patrones, con el fin de orientar la eventual aplicación de cualquiera de los cuatro modelos propuestos en las ecuaciones (4) a (7). 


\section{Estimación de los ParÁmetros del sistema logito a PARTiR DE DOS ENTRADAS}

La posibilidad de tener valores inaceptables en las probabilidades de fallecer obtenidas de los modelos descritos por las ecuaciones (4) a (7), debido a las relaciones inversas entre cocientes de mortalidad y entradas, no quedó sólo planteada a nivel teórico, ya que se obtuvo en la aplicación de dichos modelos (en varios de los 75 patrones considerados). ${ }^{11}$

Esas relaciones inversas se explican fácilmente al ver la gran variación existente en el valor del parámetro $\beta$ del sistema logito al corregir los 75 patrones.

Inicialmente podría haberse esperado, que si un patrón tuviera un valor mayor asignado a la variable esperanza de vida al nacimiento (tomada ésta como medida del nivel global del fenómeno) que en otro patrón, entonces las probabilidades de fallecer en el primero hubieran sido menores que en el segundo en todos los intervalos de edades; sin embargo, esto no ocurrió. Si se graficaran los cocientes de mortalidad de los 75 patrones en una sola figura por edades, en vez de presentarse un desplazamiento de las curvas de cocientes de mortalidad, se observaría una gran mancha que reflejaría un gran número de cruces entre las curvas de cocientes de mortalidad. De esto se concluye la existencia de relaciones inversas entre mortalidad juvenil y mortalidad adulta, y, también, la gran variación en los valores del parámetro $\beta$ en los 75 patrones: de 0.65 a 1.35.

A raíz de estos resultados, se buscó un método que permitiera estimar los parámetros $\alpha$ y $\beta$ del sistema logito a partir de dos entradas. Si bien $\alpha$ está estrechamente relacionado al nivel global de la mortalidad y $\beta$ a su comportamiento por edad, intentar deducir su relación con medidas del fenómeno para diversos intervalos de édades, resultaría un proceso de difícil solución; seguramente la asociación sería arbitraria.

El procedimiento ideado fue estimar los puntos que determinan la recta de logitos a partir de las entradas, ya que contando con dicha recta se obtienen directamente su ordenada al origen $(\alpha)$ y su dependiente $(\beta) .{ }^{12}$ Para este propósito, se tomó la variante propuesta por Brass (1973) que consiste en hacer de las diferencias de logitos (logitos observados menos logitos estándar) una función lineal de los logitos estándar; con lo cual la fórmula (1) se convierte en:

$$
D(x)=Y(x)-Y_{s}(x)=\alpha+(\beta-1) \cdot Y_{s}(x)
$$

Los puntos en el plano de logitos son las parejas ordenadas de logitos estándar y diferencias de logitos para cada edad, y, dado que el estándar es invariante, entonces sólo se requiere determinar las diferencias de logitos

11 Véase ibid., "Anexo II".

12 Véase fórmula (1). 
a partir de las entradas. Esto se obtiene al desglosar cualquier diferencia de logitos:

$$
\begin{aligned}
& D(x)=Y(x)-Y_{s}(x)=\frac{1}{2} \cdot \ln \left(\frac{1-1_{x}}{1_{x}}\right)-\frac{1}{2} \cdot \ln \left(\frac{1-1_{x}^{s}}{1_{x}^{s}}\right) \\
& =\frac{1}{2} \cdot \ln \left(\frac{\left(1-1_{x}\right) / 1_{x}}{\left(1-1_{x}^{s}\right) / 1_{x}^{s}}\right)=\frac{1}{2} \cdot \ln \left(\frac{{ }_{x} q_{o} / x p_{o}}{{ }_{x} q_{0}^{s}{ }_{x} p_{0}^{s}}\right) ;
\end{aligned}
$$

donde, en la entrada $\left({ }_{x} q_{0} / p_{x}\right) /\left({ }_{x} q_{0}^{s} / p_{0}^{s}\right)^{13}$ se mantiene además la hipótesis fundamental del sistema logito: la asociación entre un patrón observado que presenta irregularidades en su comportamiento por edad y otro bien comportado llamado estándar.

Si se toman las diferencias de logitos para todas las edades, se tiene el inconveniente práctico de requerir por lo menos tantas entradas como edades se consideren; sin embargo, buscando sólo dos puntos (el mínimo requerido para determinar una recta) se simplifica la aplicación del método. Estos dos puntos pueden obtenerse a partir del procedimiento sugerido por Brass: divídanse los puntos del plano de logitos en dos grupos de puntos contiguos, de igual número (si el total fuera par) o aproximadamente igual (si fuera non), y calcúlese el promedio aritmético de las diferencias de logitos (ordenadas) y el promedio aritmético de los logitos estándar (abscisas) en cada grupo, con lo cual se tienen los dos puntos buscados.

El problema ahora es relacionar cada una de las dos diferencias medias de logitos con las entradas. La descripción de cómo se obtuvieron estas relaciones se hará siguiendo la forma en que fueron corregidos los 75 patrones: el número de puntos, en todos los casos, fueron 16, con lo cual se tuvieron 8 puntos en cada grupo, el primero conteniendo los logitos de 0 a 35 años de edad y el segundo los logitos de 40 a 75 años. ${ }^{14}$

Tomemos la primera diferencia media de logitos (el promedio aritmético de las diferencias de logitos en el primer grupo de puntos). Esta medida está referida a la mortalidad acumulada desde el nacimiento hasta los 35 años de edad y, matemáticamente, se puede hacer igual al producto de algún factor - propio de cada edad- por la diferencia de logitos correspondiente a la misma edad. Se tomó la última diferencia, $D(35)$,

13 Se toma la expresión ${ }_{x} q_{0} / p_{0}$ en lugar de $\left(1-1_{x}\right) / 1_{x}$ con el fin de no complicar las fórmulas siguientes. Si en el sistema logito debe hacerse $1_{0}=1$, es claro entonces que $1_{x}={ }_{x} p_{0} \mathrm{y}$, por ende, que $\left(1-1_{x}\right) /\left(1_{x}={ }_{x} q_{0}{ }_{x} p_{0}\right.$.

14 Se consideró la mortalidad hasta los 75 años debido a que, como se dijo antes, las probabilidades de fallecer entre los 75 y 80 años de edad en casi todos los patrones resulta inferior a la que correspondería a la tendencia observada en los cocientes de mortalidad para edades anteriores. Al hacer referencia a estos intervalos de edades específicas, no habrá pérdida de generalidad en la descripción del método y, en cambio, ayudará a fijar mejor las ideas. 
debido a que contiene la mortalidad acumulada en todo el intervalo de edades (de 0 a 35 años):

$$
\bar{D}_{1}=\frac{\sum_{\sum}^{35} D(x)}{8}=k \cdot \cdot D(35)=k_{1} \cdot \frac{1}{2} \cdot 1 n\left(\frac{{ }_{35} q_{0} / 35 p_{0}}{{ }_{35} q_{0}^{s} /{ }_{35} p_{0}^{s}}\right) .
$$

Determinando el valor de $k_{1}$ (por ejemplo, el promedio en un gran número de casos) y mediante la ecuación (9) se puede estimar la primera diferencia media de logitos a partir de una entrada: la medida de la mortalidad acumulada entre 0 y 35 años de edad expresada por la razón ${ }_{35} q_{0} /{ }_{35} p_{0}$ en el patrón observado, ya que la misma medida en el patrón estándar es constante.

Debido a los problemas que se tienen para obtener una medida de la mortalidad desde el inicio de la vida, se creyó conveniente dividir el intervalo de 0 a 35 años, en dos intervalos: de 0 a 5 y de 5 a 35 años de edad. Partiendo de la siguiente propiedad de las probabilidades de sobrevivencia:

$$
{ }_{35} p_{0}={ }_{{ }_{5}} p_{0} \cdot{ }_{30} p_{\overline{5}},
$$

se tiene que,

$$
{ }_{3 ;} q_{0}=1-{ }_{35} p_{0}=1-{ }_{5} p_{0}{ }_{3} p_{5} ;
$$

matemáticamente, existe un número $r_{1}$ que satisface la siguiente relación:

$$
\frac{{ }_{35} q_{0}}{{ }_{33} q_{0}^{s}}=\frac{1-{ }_{5} p_{0} \cdot{ }_{30} p_{5}}{1-{ }_{5} p_{0}^{s} \cdot{ }_{30} p_{5}^{s}}=r_{1} \cdot \frac{1-{ }_{30} p_{5}}{1-{ }_{30} p_{5}^{s}}=r_{1} \cdot \frac{{ }_{30} q_{5}}{{ }_{30} q_{5}^{s}}
$$

de donde, se puede re-escribir la ecuación (9) como:

$$
\begin{aligned}
\bar{D}_{1} & =k_{1} \cdot \frac{1}{2} \cdot \ln \left(\frac{{ }_{5} p_{0}^{s}}{p_{0}} \cdot r_{1} \cdot \frac{{ }_{30} q_{5} /{ }_{30} p_{5}}{{ }_{30} q_{5}^{s} / p_{5}^{s}}\right) \\
& =\frac{k_{1}}{2} \cdot \ln \left(r_{1} \cdot \frac{p_{0}^{s}}{p_{0}}\right)+\frac{k_{1}}{2} \cdot 1 n\left(\frac{{ }_{30} q_{5} /{ }_{30} p_{5}}{{ }_{30} q_{5}^{s} /{ }_{30} p_{5}^{s}}\right),
\end{aligned}
$$

es decir, la primera diferencia media de logitos queda determinada por la mortalidad en dos intervalos de edades: antes de los 5 años y entre los 5 y 35 años.

Definiendo $R_{1}$ como:

$$
R_{1}=1 n\left(\frac{{ }_{30} q_{5} /{ }_{30} p_{5}}{{ }_{30} q_{5}^{s} /{ }_{30} p_{5}^{s}}\right)
$$


la ecuación (10) se puede escribir de manera más sencilla:

$$
\bar{D}_{1}=a_{10}+a_{11} \cdot R_{1},
$$

donde, $a_{10}$ se refiere a la parte que, en la primera diferencia media de logitos, es atribuible a la mortalidad antes de los 5 años de edad, y el coeficiente $a_{11}$ denota el cambio porcentual en la primera diferencia media de logitos debido a un cambio en la mortalidad entre los 5 y 35 años de edad.

Si la relación lineal (11) se generaliza a un gran número de casos, se estará suponiendo, entonces, que la parte debida a la mortalidad en los primeros cinco años de vida es constante en todos los casos, y que la variación en los valores de la primera diferencia media de logitos sólo estará explicada por la mortalidad entre los 5 y 35 años de edad; hecho que es deseable, ya que se quieren evitar medidas que contengan intervalos de edades desde el nacimiento. Así, con la ecuación (11) se puede estimar la primera diferencia media de logitos; los valores de $a_{10}$ y $a_{11}$, para cada sexo, fueron estimados mediante una regresión lineal simple sobre los 75 patrones y se presentan en el cuadro 5 , donde se adjunta el valor de ${ }_{30} q_{5}^{s} /{ }_{30} p_{5}^{s}$ (del estándar) necesario para calcular $\mathbf{R}_{1}$.

Para la segunda diferencia media de logitos se sigue un procedimiento análogo; existe un factor $k_{2}$ tal que:

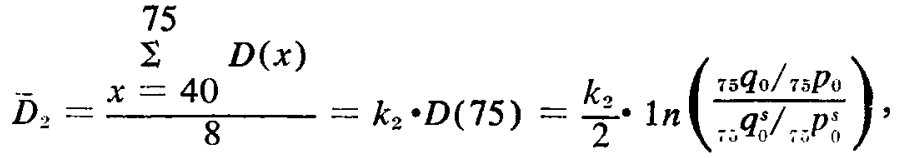

$\mathrm{y}$ dos factores $r_{2}$ y $r_{3}$ tales que satisfacen:

$$
\begin{aligned}
& \frac{{ }_{75} q_{0}}{{ }_{{ }_{55}} q_{0}^{s}}=\frac{1-{ }_{5} p_{0} \cdot{ }_{70} p_{5}}{1-{ }_{5} p_{0}^{s} \cdot{ }_{70} p_{5}^{s}}=r_{2} \cdot \frac{1-{ }_{30} p_{5} \cdot{ }_{40} p_{35}}{1-{ }_{30} p_{5}^{s} \cdot{ }_{{ }_{0} 0} p_{35}^{s}} \\
& \quad=r_{2} \cdot \frac{1-{ }_{30} p_{5}}{1-{ }_{30} p_{5}^{s}} \cdot r_{3} \cdot \frac{1-{ }_{40} p_{35}}{1-{ }_{40} p_{35}^{s}}=r_{2} \cdot \frac{{ }_{30} q_{5}}{{ }_{30} q_{5}^{s}} \cdot r_{3} \cdot \frac{{ }_{40} q_{30} q_{35}^{s}}{s}
\end{aligned}
$$

de donde, se tiene que:

$$
\begin{aligned}
& \bar{D}_{2}=\frac{k_{2}}{2} \cdot \ln \left(\frac{{ }_{5} p_{0}^{s}}{{ }_{3} p_{0}} \cdot r_{2} \cdot \frac{{ }_{30} q_{5} /{ }_{30} p_{5}}{{ }_{30} q_{\overline{5}}^{s} /{ }_{30} p_{5}^{s}} \cdot r_{3} \cdot \frac{{ }_{40} p_{35} /{ }_{35} /{ }_{40} p_{35}}{s}\right) \\
& =\frac{k_{2}}{2} \cdot \ln \left(r_{2} \cdot \frac{{ }^{\circ} p_{0}^{s}}{{ }_{\mathrm{i}} p_{0}}\right)+\frac{k_{2}}{2} \ln \left(r_{3} \cdot \frac{{ }_{30} q_{5} / 30}{{ }_{30} q_{5}^{s} /{ }_{30} p_{5}^{s}}\right) \\
& +\frac{k_{2}}{2} \cdot \ln \left(\frac{{ }_{40} q_{35} /{ }_{40} p_{35}^{s}}{{ }_{30}^{5} /{ }_{40} p_{35}^{s}}\right)
\end{aligned}
$$

y definiendo $R_{2}$ como:

$$
R_{2}=1 n\left(\frac{{ }_{40} q_{35} /{ }_{40} p_{35}}{q_{35}^{s} /{ }_{40} p_{35}^{s}}\right)
$$


Cuadro 5

Parámetros para estimar los Valores de $\alpha$ Y $\beta$ Del sistema logito ${ }^{2}$

\begin{tabular}{|c|c|c|c|c|c|c|}
\hline & & \multicolumn{5}{|c|}{ HOmbres } \\
\hline $\begin{array}{l}\text { ¿iferencia } \\
\text { de logitos }\end{array}$ & & \multicolumn{5}{|c|}{$\begin{array}{l}\text { Coeficientes de regresion para estimar } \\
\text { las diferencias medias de logitos. }\end{array}$} \\
\hline (i) & & $a_{10}$ & $a_{11}$ & & $a_{i 2}$ & $r^{2}$ \\
\hline 1 & & 0.012303 & 0.687364 & & & 0.80205 \\
\hline 2 & & 0.014899 & 0.596216 & & -0.030445 & 0.99130 \\
\hline \multicolumn{7}{|c|}{ Parámetros en el estándar mexicano } \\
\hline${ }_{30} \mathrm{q}_{5}^{\mathrm{s}} / 30^{\mathrm{P}} \mathrm{s}$ & $=$ & 0.096084 & $\bar{Y}_{1}^{\mathbf{s}}$ & $=$ & -0.915153 & \\
\hline $40^{q_{35}^{s}} / 40^{p_{35}^{s}}$ & $=$ & 1.186130 & $\overline{\mathbf{Y}}_{2}^{\mathbf{s}}$ & $=$ & -0.260060 & \\
\hline & & \multicolumn{5}{|c|}{ Mujeres } \\
\hline $\begin{array}{l}\text { diferencia } \\
\text { de logitos }\end{array}$ & . & \multicolumn{5}{|c|}{$\begin{array}{l}\text { Coeficientes de regresión para estimar } \\
\text { las diferencias medias de logitos }\end{array}$} \\
\hline (i) & & $a_{i 0}$ & $a_{i 1}$ & & $a_{i 2}$ & $x^{2}$ \\
\hline 1 & & 0.040610 & 0.715247 & & & 0.91803 \\
\hline 2 & & 0.011874 & 0.601698 & & -0.043045 & 0.99470 \\
\hline \multicolumn{7}{|c|}{ Parámetros en el estánđar mexicano } \\
\hline $30^{\mathrm{q}}{ }_{5}^{\mathrm{S}} / 30^{\mathrm{P}} \mathrm{S}$ & $=$ & 0.073189 & $\overline{\mathbf{y}_{I}} \mathbf{s}$ & $=$ & -1.002043 & \\
\hline $40^{q_{35}^{5} / 40^{P_{35}^{5}}}$ & $=$ & 0.953107 & $\bar{Y}_{2}^{\mathrm{s}}$ & $=$ & -0.377993 & \\
\hline
\end{tabular}

1 Para su aplicación, véanse las fórmulas (11), (12) y el texto.

se tiene finalmente:

$$
\bar{D}_{2}=a_{20}+a_{21} \cdot R_{1}+a_{22}{ }_{1} R_{2},
$$

donde, $a_{20}$ se refiere a la parte que, en la segunda diferencia media de logitos, es atribuible a la mortalidad en los primeros cinco años de vida, el coeficiente $a_{21}$ denota el cambio porcentual en la segunda diferencia media de logitos debido a un cambio en la mortalidad entre los 5 y 35 años de edad, y el coeficiente $a_{22}$ denota el cambio porcentual en la segunda diferencia media de logitos debido a un cambio en la mortalidad entre los 35 y 75 años de edad.

Si la relación (12) se generaliza a un gran número de casos, se estará suponiendo, entonces, que la segunda diferencia media de logitos sólo varía por cambios en la mortalidad a partir de los 5 años de edad. Los coeficientes de la ecuación (12) fueron obtenidos mediante regresiones dobles sobre los 75 patrones, y, con el valor de ${ }_{40} q_{35}^{s} /{ }_{40} p_{35}^{s}$, se presentan en el cuadro 5 .

Los valores obtenidos para $a_{10}$ y $a_{20}$-términos (o coeficientes) independientes en las ecuaciones (11) y (12) - fueron bastante bajos (véase 
el cuadro 5); esto indica que la variación en las dos diferencias medias de logitos está explicada, casi totalmente, por la mortalidad a partir de los 5 años de edad; con lo cual, se pueden esperar estimaciones muy precisas de estas diferencias, sin considerar en su determinación la mortalidad en los primeros cinco años de vida, y con los logitos medios estándar aplicar el método logito y obtener un patrón modelo adecuado al caso particular que se esté estudiando.

\section{Patrones modelo obtenidos mediante interpolación en tablas}

En varios casos el patrón modelo correspondiente difirió notoriamente del patrón observado, cuando se aplicó el último método (estimación de los parámetros del sistema logito a partir de dos entradas) a los 75 patrones considerados.

Si bien se puede concluir - de la aplicación de los modelos desarrollados en los dos incisos anteriores a los 75 patrones - que aquellos que relacionan los logaritmos de los cocientes de mortalidad con los logaritmos de las entradas ${ }_{30} q_{5}$ y ${ }_{40} q_{35}$, representados por la ecuación (7), fueron los mejores, una posibilidad nada remota, (como ocurrió en algunos casos) es obtener ciertos valores de las entradas que proporcionen probabilidades de fallecer inaceptables para la realidad mexicana, dadas las relaciones inversas que se presentaron en este tipo de modelos. Si esto último llegara a ocurrir, el usuario debería verificar la bondad del ajuste entre el patrón observado y el patrón modelo, pudiendo llegar a ser tan compleja la aplicación (por ejemplo, encadenándola para una convergencia o comparando resultados de la aplicación de los diferentes modelos propuestos), que la utilidad misma de los modelos quedaría en entredicho.

Buscando una aplicación más satisfactoria y sobre todo más sencilla, un último procedimiento consistió en obtener un gran número de patrones aplicando el sistema logito para diversas combinaciones de $\alpha$ y $\beta$ con los estándares mexicanos, extrayendo en cada uno las probabilidades de fallecer ${ }_{10} q_{5},{ }_{20} q_{45},{ }_{30} q_{5}$ y ${ }_{40} q_{35}$, de tal manera que con éstas y sus correspondientes probabilidades en un patrón observado, mediante interpolación se determine el patrón modelo apropiado al caso en estudio.

Después de la interpolación es lógico esperar que se mantengan los valores de las entradas en el patrón modelo ajustado, debido a lo cual me inclino más por este último método que por cualquiera de los desarrollados en los dos incisos anteriores; quedando como otra alternativa la aplicación directa del método logito con los estándares mexicanos, aunque este último procedimiento representa un proceso algo más largo y laborioso que la interpolación en un gran conjunto de patrones modelo.

Ese gran número de patrones fueron generados para valores de $\alpha$ y $\beta$ en intervalos un poco más amplios que los obtenidos al aplicar el sistema logito a los 75 patrones considerados: se variaron los valores de ambos 
parámetros en $0.05, \alpha$ de -0.50 a 0.55 y $\beta$ de 0.75 a 1.25 , resultando así 264 patrones modelo para cada sexo.

Para su uso, se propone la interpolación en los cocientes ${ }_{10} q_{5}$ y ${ }_{20} q_{45} \mathrm{o}$ en los cocientes ${ }_{30} q_{5}$ y ${ }_{40} q_{35}$, aunque se pueden elegir cualesquiera otras entradas, incluso más de dos. ${ }^{15}$

\section{Aplicación de los MOdelos obtenidos a los patrones observados} en BaJa California SUR en 1964-1966

La elección de los patrones observados en Baja California Sur en el trienio 1964-1966 para ejemplificar la aplicación de los patrones modelo de mortalidad obtenidos para México, responde a dos razones: a) los mismos no fueron considerados en la construcción de los modelos y $b$ ) Baja California Sur y Quintana Roo, son las entidades federativas que presentan mayores irregularidades en su comportamiento por edad.

Las probabilidades de fallecer, a partir de la edad exacta 1 , fueron obtenidas de las tasas centrales observadas en el trienio y de la fórmula encontrada por Reed y Merrell (1939); donde, la tasa central ${ }_{4} M_{1}$ fue obtenida de diagramas de Lexis construidos con las estadísticas vitales. La probabilidad de fallecer en el primer año de vida fue hecha igual a la tasa de mortalidad infantil observada en el trienio.

Los resultados aparecen en el cuadro A.I.1 para hombres y en el cuadro A.I.2 para mujeres (columna "observados").

El primer tipo de modelos que se obtuvo para estos patrones fue la aplicación del sistema logito, lo cual se hizo tomando los dos puntos que resultan de considerar los logitos medios observados y los logitos medios estándar en dos grupos en que se dividieron los puntos del plano de logitos. Los puntos considerados, al igual que en la corrección de lós 75 patrones para la construcción de los modelos, fueron 16 (para las edades $1,5,10$, $15,20, \ldots, 70$ y 75), y los valores de los parámetros $\alpha$ y $\beta$ fueron -0.266460 y 0.992479 , respectivamente, para hombres y -0.349533 y 0.736747 para mujeres. Los patrones modelo resultantes aparecen en los cuadros A.I.1 y A.I.2 (columna "logito").

Debido a que al aplicar el modelo que relaciona probabilidades de fallecer con los cocientes de mortalidad ${ }_{10} q_{5}$ y ${ }_{20} q_{45}$ en la ecuación (4) proporcionó una probabilidad de fallecer entre las edades exactas 1 y 5 años $\left({ }_{1} q_{1}\right)$ negativa en el caso masculino (-3.42 por mil), se dejaron de lado este tipo de modelos así como los que relacionan las probabilidades de fallecer con los cocientes ${ }_{30} q_{\overline{5}} \mathrm{y}_{40} q_{35}$ (los de la ecuación (6)), para ambos sexos.

15 Los patrones modelo obtenidos para cada sexo y las entradas correspondientes para interpolar, pueden verse en el Anexo III de Partida (1980), pp. 172-205. 
Un segundo tipo de modelos aplicados a Baja California Sur alrededor de 1965 son aquellos que relacionan los logaritmos decimales de las probabilidades de fallecer con los logaritmos decimales de los cocientes ${ }_{10} q_{5}$ $\mathrm{y}_{20} q_{45}$, cuyos resultados aparecen en la columna "Modelo 1" de los cuadros A.I.1 y A.I.2; donde, por ejemplo, para obtener el cociente de mortalidad ${ }_{5} q_{20}$ masculino se aplica la ecuación:

$$
{ }_{5} Z_{20}=-0.30383+0.83652 \cdot{ }_{10} Z_{5}+0.20612 \cdot{ }_{20} Z_{45},
$$

\section{Cuadro A.I.1}

Probabilidades de fallecer en los patrones masculinos, OBSERVADO Y MODELOS CORRESPONDIENTES, EN BAJA CALIFORNIA SUR, 1964-1966

(Por mil)

\begin{tabular}{|c|c|c|c|c|c|c|}
\hline Edad & Observado & Logito & Modelo 1 & Modelo 2 & Mode Io 3 & Interpolacion \\
\hline 0 & 57.92 & 52.10 & 15.49 & 44.57 & 35.64 & 32.82 \\
\hline 1 & 20.04 & 22.79 & 4.01 & 11.31 & 17.89 & 17.73 \\
\hline 5 & 2.37 & 6.38 & 3.16 & 5.11 & 5.14 & 5.17 \\
\hline 10 & 3.14 & 4.52 & 2.33 & 3.67 & 3.67 & 3.71 \\
\hline 15 & 5.27 & 6.85 & 3.69 & 5.65 & 5.62 & 5.70 \\
\hline 20 & 12.90 & 10.74 & 6.14 & 9.06 & 8.93 & 9.12 \\
\hline 25 & 11.86 & 13.33 & 8.21 & 11.56 & 11.26 & 11.60 \\
\hline 30 & 13.73 & 15.66 & 10.44 & 13.97 & 13.46 & 13.98 \\
\hline 35 & 18.00 & 19.10 & 14.14 & 17.58 & 16.72 & 17.52 \\
\hline 40 & 18.48 & 23.57 & 18.64 & 22.41 & 21.05 & 22.27 \\
\hline 45 & 33.87 & 30.06 & 26.13 & 29.56 & 27.44 & 29.32 \\
\hline 50 & 44.37 & 39.44 & 37.95 & 40.20 & 36.89 & 39.81 \\
\hline 55 & 58.22 & 53.69 & 57.59 & 56.95 & 51.61 & 56.26 \\
\hline 60 & 76.49 & 76.00 & 91.20 & 83.79 & 75.28 & 22.85 \\
\hline 65 & 120.64 & 111.64 & 149.78 & 127.49 & 114.24 & 126.65 \\
\hline 70 & 211.18 & 176.21 & $176: 21$ & 259.72 & 206.90 & 206.91 \\
\hline 75 & 356.59 & 300.07 & 462.80 & 352.97 & 325.61 & 357.61 \\
\hline 80 & 343.28 & 549.44 & 791.87 & 621.29 & 594.93 & 631.89 \\
\hline${ }_{10} q_{5}$ & 5.50 & 10.87 & 5.48 & 8.76 & 8.79 & 8.86 \\
\hline $30 q_{5}$ & 48.33 & 56.17 & 33.52 & 48.07 & 47.16 & 48.32 \\
\hline $20^{9} 45$ & 197.00 & 185.34 & 197.57 & 195.22 & 178.53 & 193.27 \\
\hline $40^{q_{35}}$ & 463.13 & 428.99 & 511.37 & 465.15 & 430.11 & 463.24 \\
\hline $70^{e} \frac{a f}{5}$ & 61.983 & 61.728 & 61.581 & 61.960 & 62.39 .8 & 61.973 \\
\hline$e \frac{a /}{5}$ & 65.253 & 65.927 & 64.123 & 65.476 & 66.329 & 65.466 \\
\hline
\end{tabular}

a En el cálculo de ambas esperanzas de vida se utilizó la fórmula: $L_{x}=\frac{n}{2}\left(1_{x}+1_{x+n}\right)$

para todas las edades, excepto en el último grupo, donde se utilizó la fórmula encontrada por Rodolfo Corona (1972): $L_{85+}=-11672.27+6.23 \cdot 1_{85}$ con $1_{0}=100,000$. 
Cuadro A.I.2

Probabilidades de fallecer en los patrones femeninos, obSERVADO Y MODElos CORRESPONDIENTES, EN BAJA CaLIFoRNIA SuR, 1964-1966

(Por mil)

\begin{tabular}{|c|c|c|c|c|c|c|}
\hline Edad & Observado & Logito & Modelo 1 & Modelo 2 & Modelo 3 & Interpolacion \\
\hline 0 & 53.47 & 67.73 & 27.44 & 47.71 & 32.94 & 41.99 \\
\hline 1 & 19.70 & 26.09 & 10.19 & 18.75 & 20.64 & 22.12 \\
\hline 5 & 3.53 & 6.32 & 4.68 & 5.67 & 5.49 & 5.67 \\
\hline 10 & 4.03 & 3.75 & 2.88 & 3.42 & 3.34 & 3.42 \\
\hline 15 & 3.48 & 4.89 & 3.87 & 4.51 & 4.45 & 4.52 \\
\hline 20 & 8.60 & 7.40 & 6.12 & 6.94 & 6.92 & 6.96 \\
\hline 25 & 10.72 & 9.49 & 8.28 & 9.11 & 9.21 & 9.13 \\
\hline 30 & 10.63 & 11.43 & 10.60 & 11.26 & 11.57 & 11.29 \\
\hline 35 & 18.21 & 13.71 & 13.63 & 13.87 & 14.53 & 13.94 \\
\hline 40 & 14.82 & 16.40 & 17.53 & 17.10 & 18.27 & 17.20 \\
\hline 45 & 24.47 & 19.99 & 23.09 & 21.56 & 23.47 & 21.67 \\
\hline 50 & 35.83 & 25.42 & 31.95 & 28.33 & 31.61 & 28.58 \\
\hline 55 & 48.06 & 34.92 & 48.14 & 40.54 & 46.29 & 40.90 \\
\hline 60 & 66.75 & 47.03 & 71.78 & 57.07 & 66.80 & 57.61 \\
\hline 65 & 77.20 & 67.48 & 114.08 & 85.74 & 102.85 & 86.66 \\
\hline 70 & 123.20 & 104.28 & 192.77 & 138.29 & 169.38 & 140.14 \\
\hline 75 & 201.73 & 177.51 & 343.44 & 241.24 & 297.89 & 245.96 \\
\hline 80 & 313.08 & 348.92 & 621.95 & 459.12 & 549.34 & 471.72 \\
\hline${ }_{10} q_{5}$ & 7.55 & 10.05 & 7.55 & 9.07 & 8.81 & 9.07 \\
\hline $30^{q}{ }_{5}$ & 40.32 & 42.53 & 35.90 & 40.24 & 40.31 & 40.32 \\
\hline $20^{q_{45}}$ & 164.39 & 121.60 & 164.44 & 139.88 & 158.36 & 141.01 \\
\hline $40^{q_{35}}$ & 346.05 & 288.22 & 420.94 & 343.20 & 393.22 & $346 \because \therefore$ \\
\hline $70^{\mathrm{e}^{\mathrm{a} /}}$ & 63.367 & 64.085 & 63.357 & 63.743 & 63.265 & 62.708 \\
\hline$e_{0}^{a}$ & 69.970 & 71.215 & 67.511 & 69.593 & 67.985 & 69.393 \\
\hline
\end{tabular}

a En el cálculo de ambas esperanzas de vida se utilizó la fórmula:

$L_{x}=\frac{n}{2}\left(1_{x}+1_{x+n}\right)$

para todas las edades, excepto en el último grupo, donde se utilizó la fórmula encontrada por Rodolfo Corona (1972): $L_{85+}=-11672.27+6.23 \cdot 1_{85}$ con $1_{0}=100,000$.

donde, ${ }_{n} Z_{x}$ es el logaritmo decimal de $1000{ }_{n} q_{x}$ y los coeficientes están tomados del cuadro 2 .

El cociente de mortalidad ${ }_{10} q_{\overline{5}}$ masculino fue igual a 5.50 por mil y el cociente ${ }_{20} q_{45}$ igual a 197 por mil en Baja California Sur en 1964-1966, de donde, ${ }_{10} Z_{5}=0.74036$ y ${ }_{20} Z_{45}=2.29447 \mathrm{y}$, resolviendo la ecuación anterior, se tiene que ${ }_{5} Z_{20}=0.78843 \mathrm{y}$, finalmente, extrayendo antilogaritmo se tiene que: 
${ }_{5} q_{20}={ }^{\prime \prime} 0.78843=6.14$ por mil.

Un tercer tipo de modelos aplicados para este ejemplo fueron aquéllos que consideran relacionados los logaritmos de los cocientes de mortalidad con los logaritmos decimales de las probabilidades de fallecer ${ }_{30} q_{5}$ y ${ }_{40} q_{35}$, cuyos resultados aparecen en la columna "Modelo 2" de los cuadros A.I.1 y A.I.2.

También fue aplicado a los patrones observados en Baja California Súr alrededor de 1965 el método que consiste en obtener los parámetros $\alpha$ y $\beta$ del sistema logito habiendo estimado las diferencias medias de logitos.

Los valores de $R_{1}$ y $R_{2}$ para el ejemplo fueron:

$$
\begin{aligned}
R_{1} & =\ln \left(\left({ }_{3 i} q_{5} /{ }_{30} p_{5}\right) /\left({ }_{30} q_{55}^{s} /{ }_{30} p_{5}^{s}\right)\right)=\ln (0.050784 / 0.096084) \\
& =-0.637641, \mathrm{y}, \\
R_{2} & =\ln \left({\left.\left.\left({ }_{40} q_{35} /{ }_{40} p_{35}\right) /{ }_{4} q_{45}^{s} /{ }_{40} p_{535}^{s}\right)\right)=1 n(0.862648) /(1.186130)}=-0.318444,\right.
\end{aligned}
$$

para hombres, y

$$
\begin{aligned}
& R_{1}=1 n(0.042014 / 0.073189)=-0.555042, \mathrm{y}, \\
& R_{2}=\ln (0.529169 / 0.953107)=-0.558420,
\end{aligned}
$$

para mujeres; donde $s$ denota la medida en el estándar mexicano correspondiente (véase el cuadro 5). Entonces, las diferencias medias de logitos son, para hombres:

$$
\begin{aligned}
& \bar{D}_{1}=0.012303+0.687364 \cdot R_{1}=-0.425988, \mathrm{y}, \\
& \bar{D}_{2}=0.014899+0.596216 \cdot R_{1}-0.030445 \cdot R_{2}=-0.355578 ; \mathrm{y},
\end{aligned}
$$

para mujeres:

$$
\begin{aligned}
& \widetilde{D}_{1}=0.040610+0.715247 \cdot R_{1}=-0.356382, \mathrm{y} \\
& \bar{D}_{2}=0.011874+0.601698 \cdot R_{1}-0.043045 \cdot R_{2}=-0.298056 .
\end{aligned}
$$

De la fórmula (8) se puede ver fácilmente que:

$$
\bar{Y}_{i}=\bar{D}_{i}+\bar{Y}_{i}^{s},
$$

donde, $\bar{Y}$ es el logito medio del i-ésimo grupo de puntos $(i=1,2)$ y $s$ denota el estándar, con lo cual, se tiene que, para hombres: 


$$
\bar{Y}_{1}=-1.341141 \text { y } \bar{Y}_{2}=-0.615638, \mathrm{y},
$$

para mujeres:

$$
\bar{Y}_{1}=-1.358425 \text { y } \bar{Y}_{2}=-0.676049,
$$

$\mathrm{y}$, finalmente, resolviendo el siguiente sistema de ecuaciones simultáneas (véase la ecuación (1)):

$$
\begin{aligned}
& \bar{Y}_{1}=\alpha+\beta \cdot \bar{Y}_{1}^{s} \\
& \bar{Y}_{2}=\alpha+\beta \cdot \bar{Y}_{2}{ }^{s}
\end{aligned}
$$

se obtienen los valores de $\alpha$ y $\beta$ y se aplica el sistema logito. Los valores de $\alpha$ y $\beta$ fueron, para hombres:

$$
\alpha=-0.327627 \text { y } \beta=1.107481, \mathrm{y} \text {, }
$$

para mujeres:

$$
\alpha=-0.262727 \text { у } \beta=1.093464 \text {. }
$$

Los resultados de la aplicación de este método al ejemplo considerado aparecen en la columna "Modelo 3" de los cuadros A.I.1 y A.I.2. Se podrá ver que los valores de $\alpha$ y de $\beta$ difieren sensiblemente de los obtenidos al aplicar directamente el sistema logito a los patrones observados; sin embargo, analizando las medidas de ajuste en los cuadros A.I.1 y A.I.2, se puede concluir que los modelos obtenidos por este último método resultaron ser mejores que los obtenidos al aplicar directamente el sistema logito.

Finalmente, se aplicó el procedimiento basado en interpolación sobre un gran número de patrones modelo con dos entradas. Una forma de interpolación doble (pues se consideran dos entradas) es la siguiente: se requieren cuatro patrones modelo para la interpolación, digamos el 1 , el 2 , el 3 y el 4 , y denotemos por $e_{i 1}$ la primera entrada $\left({ }_{10} q_{5} 0{ }_{30} q_{5}\right)$ y por $e_{i 2}$ la segunda entrada $\left({ }_{20} q_{45} \mathrm{O}{ }_{40} q_{35}\right)$ en el i-ésimo de estos patrones. El esquema que se tiene es:

$$
e_{11}
$$

$e_{12}$

(2)

$$
e_{21}
$$

$e_{22}$ $e_{31}$

$e_{32}$

(4)

$e_{41}$

$e_{42}$ con: $\quad e_{11} \leqq E_{1} \leqq e_{21}$,

$e_{31} \leqq E_{1} \leqq e_{41}$,

$$
\begin{aligned}
& e_{12} \leqq E_{2} \leqq e_{22}, \\
& e_{22} \leqq E_{2} \leqq e_{42}, y,
\end{aligned}
$$


$E_{1}$ y $E_{2}$ son la primera y segunda entrada, respectivamente, en el patrón observado; el cual nos garantiza que no habrá extrapolaciones, sólo interpolaciones, y con esto, que obtendremos resultados más precisos.

Interpolando con la primera entrada entre el patrón (1) y el (2) y entre los patrones (3) y (4), tendremos dos patrones modelo resultantes, digamos el $a$ y el $b$, con la misma primera entrada y con la segunda entrada cubriendo a la observada, es decir, el esquema es:

(a)

(b)

$e_{a_{1}} \quad e_{b_{1}}$

con: $e_{a_{1}}=e_{b_{1}}=E_{1}, \mathrm{y}$,

$e_{a 2} \quad e_{b_{2}}$

$e_{a 2}<E_{2}<e_{b_{2}}$.

Finalmente, interpolando con la segunda entrada en los patrones $(a)$ y (b) se tiene el patrón modelo correspondiente al patrón observado. Denotando por $z_{1}$ la pendiente en la interpolación lineal con los patrones modelo (1) y (2) y por $z_{2}$ la pendiente en la interpolación lineal con los patrones (3) y (4) con la primera entrada, tenemos que, cualquier probabilidad de fallecer en los patrones $(a)$ y $(b)$ quedará dada por:

$$
\begin{aligned}
& { }_{n} q_{x}^{a}=\left(1-z_{1}\right) \cdot{ }_{n} q_{x}^{1}+z_{1} \cdot{ }_{n} q_{x}^{2}, \mathrm{y}, \\
& { }_{n} q_{x}^{b}=\left(1-z_{2}\right) \cdot{ }_{n} q_{x}^{3}+z_{2} \cdot{ }_{n} q_{x}^{4},
\end{aligned}
$$

donde ${ }_{n} q_{x}^{i}$ es la probabilidad de fallecer entre las edades exactas $x$ y $x+n$ en el i-ésimo patrón modelo; $\mathrm{y}$, después de la interpolación lineal con la segunda entrada, siendo $z_{3}$ la pendiente, se tiene que en el patrón modelo resultante cualquier probabilidad de fallecer queda dada por:

$$
\begin{aligned}
{ }_{x} q_{x} & =\left(1-z_{3}\right) \cdot{ }_{n} q_{x}^{a}+z_{3} \cdot{ }_{n} q_{x}^{b} \\
& =\left(1-z_{1}\right)\left(1-z_{3}\right) \cdot{ }_{n} q_{x}^{1}+z_{1}\left(1-z_{3}\right) \cdot{ }_{n} q_{x}^{2}+z_{3}\left(1-z_{2}\right) \cdot{ }_{n} q_{x}^{3} \\
& +z_{2} \cdot z_{3} \cdot{ }_{n} q_{x}^{4},
\end{aligned}
$$

es decir, se tiene una ecuación con los cuatro patrones modelo considerados inicialmente para la interpolación, cada uno de ellos con un ponderador asociado; de donde, se tiene entonces que cualquier probabilidad de fallecer en el patrón modelo correspondiente al observado queda dada por:

$$
{ }_{n} q_{x}=w_{1} \cdot{ }_{n} q_{x}^{1}+w_{2} \cdot{ }_{n} q_{x}^{2}+w_{3} \cdot{ }_{n} q_{x}^{3}+w_{4} \cdot{ }_{n} q_{x}^{4},
$$

con:

$$
\begin{aligned}
& w_{1}=\left(1-z_{1}\right)\left(1-z_{3}\right), \\
& \boldsymbol{w}_{2}=z_{1} \cdot\left(1-z_{3}\right), \\
& \boldsymbol{w}_{3}=z_{3} \cdot\left(1-z_{2}\right),
\end{aligned}
$$




$$
\begin{aligned}
& w_{4}=z_{2} \cdot z_{3} \\
& z_{1}=\left(E_{1}-e_{11}\right) /\left(e_{21}-e_{11}\right), \\
& z_{2}=\left(E_{1}-e_{31}\right) /\left(e_{41}-e_{31}\right), \\
& z_{3}=\left(E_{2}-e_{a 2}\right) /\left(e_{b 2}-e_{a 2}\right), \\
& e_{a 2}=e_{12}+z_{1}\left(e_{22}-e_{21}\right), \mathrm{y}, \\
& e_{b 2}=e_{32}+z_{2}\left(e_{42}-e_{32}\right) .
\end{aligned}
$$

Con el fin de obtener un patrón modelo lo más preciso posible, deben seleccionarse estos cuatro patrones que servirán para la interpolación más próxima a las entradas $\left(E_{1}\right.$ y $\left.E_{2}\right)$ en el patrón observado.

Para el patrón masculino y para el femenino de Baja California Sur alrededor de 1965, se eligieron como entradas las probabilidades de fallecer ${ }_{30} q_{5} \mathrm{y}_{40} q_{45}$, debido a que considerando como entrada ${ }_{10} q_{5}$ se tiene sobremortalidad femenina y ello podría arrojar un inadecuado diferencial por sexo en los resultados.

El esquema queda entonces planteado (véanse las tablas del "Anexo III" de Partida (1980)),

para hombres:

(1)

48.2124

452.0516

(2)

49.8071

440.6136
46.5631

463.1173

(4) $\mathrm{y}$, para mujeres:

(1)

39.4420

322.2701

43.1218

342.0517
(3)

38.9686

369.8341

pues, en el caso masculino $E_{1}=48.3335$ y $E_{2}=463.1252$, y, para el caso femenino $E_{1}=40.3190$ y $E_{2}=346.0577$. Con estos datos, se tiene entonces que,

$$
\begin{aligned}
& \text { para hombres: } \\
& z_{1}=0.075939 \\
& z_{2}=0.401561 \\
& e_{a 2}=451.1830 \\
& e_{b_{2}}=472.1737 \\
& z_{3}=0.568552 \\
& w_{1}=0.398684 \\
& w_{2}=0.032764 \\
& w_{3}=0.340244 \\
& w_{4}=0.228308
\end{aligned}
$$

y, para mujeres:

$$
\begin{aligned}
& z_{1}=0.238328 \\
& z_{2}=0.922530 \\
& e_{a 2}=326.9846 \\
& e_{b_{2}}=361.7328 \\
& z_{3}=0.548895 \\
& w_{1}=0.343594 \\
& w_{2}=0.107511 \\
& w_{3}=0.042523 \\
& w_{4}=0.506372,
\end{aligned}
$$

donde, se puede ver que la suma de los cuatro ponderadores $\left(w_{1}, w_{2}, w_{3}\right.$ y $w_{4}$ ) es igual a uno, hecho indispensable en cualquier interpolación. 
Gráfica A.I.1

Probabilidades de fallecer en los patrones masculinos, observado Y MODElos CORResPondientes, en Baja California Sur, 1964-1966

(Por mil)

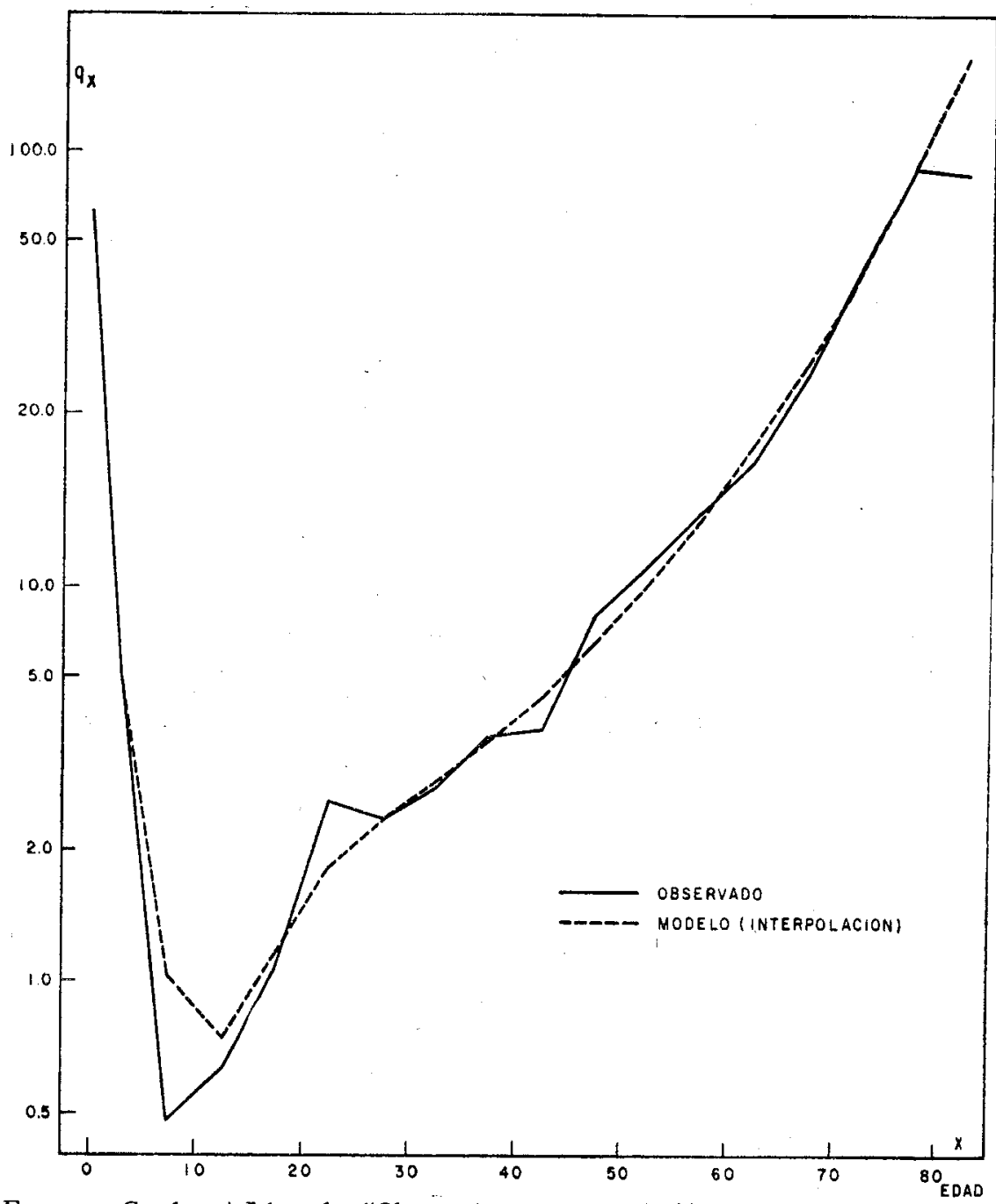

Fuente: Cuadro A.I.1, cols. "Observado" e "Interpoiación". 


\section{Gráfica A.I.2}

Probabilidades DE FALLECER EN LOS PATRONES FEMENINOS oBsERVADO Y MODElos CORRESPONDIENTES, EN BAJA CALIFornia SUR, 1964-1966

(Por mil)

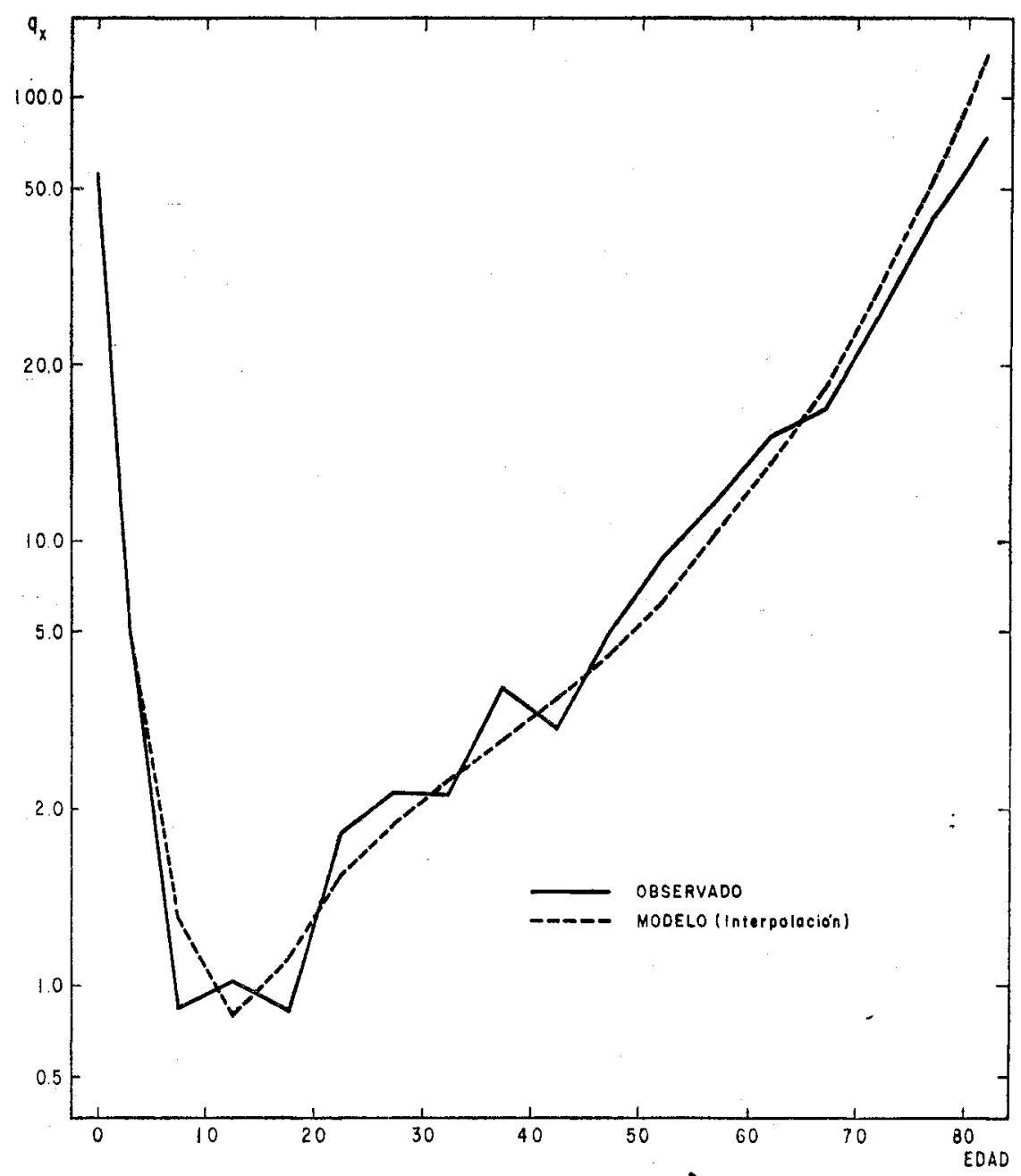

Fuente: Cuadro A.I.2, cols. "Observado" e "Interpolación". 
Los resultados de este método aparecen en la columna "Interpolación" de los cuadros A.I.1 y A.I.2; donde, por ejemplo, el cociente femenino ${ }_{5} \boldsymbol{q}_{35}$ en el patrón modelo fue obtenido de la ecuación:

$$
\begin{aligned}
{ }_{5} q_{35} & =0.343594 \cdot{ }_{5} q_{35}^{1}+0.107511 \cdot{ }_{5} q_{35}^{2}+0.042523 \cdot{ }_{5} q_{35}^{3} \\
& +0.506372{ }_{5} q_{35}^{4}=0.343594(0.013398)+0.107511(0.014629) \\
& +0.042523(0.013869)+0.506372(0.014163)=0.01394 \\
& =13.94 \text { por mil. }
\end{aligned}
$$

Como prueba de ajuste se proporcionan en los cuadros A.I.1 y A.I.2 ios cocientes de mortalidad ${ }_{10} q_{5},{ }_{30} q_{5},{ }_{20} q_{45}$ y ${ }_{40} q_{35}$, la esperanza de vida parcial entre 5 y 75 años de edad y la esperanza de vida al nacimiento. De estos resultados, los modelos obtenidos por interpolación se consideraron los mejores, pues presentan las mejores estimaciones en conjunto. Finalmente, hay que notar que las probabilidades de fallecer en el primer año de vida $\left({ }_{1} q_{0}\right)$ resultaron menores que las observadas y que el valor de ${ }_{4} q_{1}$ se aleja algo de su valor observado. Como alternativa de corrección, se propone asignar el cociente observado ${ }_{4} q_{1}$ al patrón modelo obtenido por interpolación, ya que el obtenido por el modelo se aleja más de un $10 \%$ del observado; ${ }^{16} \mathrm{y}$, dado que al aplicar las relaciones (2) a los valores observados de ${ }_{4} q_{1}$ se obtienen valores inferiores a los observados en las ${ }_{1} q_{0}$, se propone asignar las probabilidades de fallecer en el primer año de vida observadas al patrón modelo obtenido por interpolación, quedando así el patrón modelo definitivo correspondiente a Baja California Sur 1964-1966.

\section{Conclusiones}

1. Después de una breve evaluación de la calidad de la fuente de información de los 75 patrones observados en la República Mexicana, considerados para la construcción de los modelos, se concluyó que éstos podrían referirse, de manera muy aproximada, a las poblaciones residentes en las diferentes entidades federativas del país, e, incluso, a cualquier división geográfica mayor.

2. De un análisis del comportamiento de la mortalidad antes de los 5 años de edad, observado en los resultados de las historias de embarazos de la Encuesta Mexicana de Fecundidad y en los diferentes modelos de mortalidad construidos hasta ahora, se concluyó que era necesario corregir la subestimación observada en la probabilidad de fallecer en el

${ }_{16}$ En el inciso 3 (p. 10) se explica la forma de seleccionar el valor de ${ }_{4} q_{1}$. 
primer año de vida $\left({ }_{1} q_{0}\right)$ en base a los resultados de dicha Encuesta, a partir del valor observado del cociente de mortalidad ${ }_{4} q_{1}$.

3. Para corregir las irregularidades observadas en el comportamiento por edad en los 75 casos considerados, se eligió el método logito de Brass, surgiendo la necesidad de construir dos estándares mexicanos (uno para cada sexo); con lo cual se tuvo un primer tipo de modelos: la aplicación del sistema logito con estos estándares.

4. Con el fin de obtener patrones modelo de manera más sencilla que los que resultan de la aplicación del sistema logito, se desarrollaron dos metodologías. La primera consistió en relacionar, de diversas formas, probabilidades de fallecer $\left({ }_{n} q_{x}\right)$ con dos medidas resumidas del fenómeno o entradas. La segunda consistió en determinar los parámetros $\alpha$ y $\beta$ del sistema logito en base a la estimación de diferencias medias de logitos a partir de dos entradas.

5. Al aplicar estas dos metodologías a los 75 patrones considerados, se observó que, en varios casos, los resultados no fueron satisfactorios (el patrón modelo fue notoriamente diferente del patrón observado); por lo cual se desarrolló una última metodología que consistió en generar un gran número de patrones, aplicando el sistema logito para diversas combinaciones de los parámetros $\alpha$ y $\beta$, extrayendo de cada uno diversas entradas con las cuales, mediante interpolación, se determinó el patrón modelo. Este último método fue considerado el mejor, debido a que mantiene los valores de las entradas del patrón modelo.

6. Finalmente, debe tenerse en cuenta que, aun cuando se lleve a cabo el método de interpolación entre un gran número de patrones modelo, pueden resultar valores inaceptables en las probabilidades de fallecer en los primeros cinco años de vida $\left({ }_{1} q_{0} \mathrm{y}_{4} q_{1}\right)$, cuya corrección debe ser hecha de alguna forma, y aquí se propone que sea la misma que se siguió en la corrección de los 75 patrones considerados.

\section{BIBLIOGRAFIA}

Arriaga, Eduardo (1968), New life tables for Latin American Populations in the nineteenth and twentieth Centuries. University of California Press, Berkeley. (Populations Monograph Series 3.)

Barral Souto, J. y Somoza, J. (1953), "Construcción de una tabla abreviada de mortalidad para la Argentina", Segundo Coloquio de Estadistica. Córdoba, Argentina.

Benítez, Raúl y Cabrera, Gustavo (1967), Tablas abreviadas de mortalidad de la población de México 1930, 1940, 1950 y 1960. El Colegio de México, México.

Bourgeois-Pichat, Jean (1962), "Análisis factorial de las tasas de mortalidad por edad y por sexo: contribución al estudio de las dimensiones 
de la mortalidad" en Boletín de Población de las Naciones Unidas No. 6, Nueva York.

(1970), "Las tablas modelo de mortalidad" en El concepto de población estable. Naciones Unidas, Nueva York.

Brass, William (1973), "El sistema logito" y "Fundamentos y propiedades del sistema logito; uso del sistema logito" en Seminario sobre métodos para medir variables demográficas (fecundidad y mortalidad). CELADE, Santiago de Chile.

(1974), "Sobre la escala de la mortalidad" en Métodos para estimar la fecundidad y la mortalidad en poblaciones con datos limitados. CELADE, Santiago de Chile, pp. 135-180.

Cabrera, G., Ordorica, M. y García, L. (1973), "Tabla abreviada de mortalidad de México, 1969-1971". CEED, El Colegio de México, México. Inédito.

Coale, Ansley y Demeny, Paul (1966), Model regional life tables and stable populations. Princeton. University Press, Princeton.

Cordero, Eduardo (1968), "La subestimación de la mortalidad infantil en México" en Demografía y Economía II (1), 4. pp.

Carrier, Norman y Hobcraft, John (1975), Estimaciones demográficas para sociedades en desarrollo. CELADE, San José, Costa Rica.

Corona, Rodolfo (1972), "Determinación de la mortalidad por medio de técnicas de William Brass con especial referencia al sistema logito".

CELADE, Santiago de Chile.

Gabriel, K. R. y Ronen, Ilána (1958), "Estimates of mortality from infant mortality rates" en Population Studies XII (2), pp. 164-169.

Ledermann, Sully (1969), Nouvelles tables-type de mortalité. INED, Paris. (Cahier 53.)

y Breas, Jean (1959), "Les dimensions de la mortalité" en Population 14 (4), pp. 653-659.

Naciones Unidas (1963), Modelos de mortalidad por sexo y edad. Nueva York.

Ordorica, Manuel y otros (1975), Evaluación de la mortalidad infantil en la República Mexicana 1930-1970. SIC, DGE, México. (Evaluación y análisis III-1.)

(1976), Tablas abreviadas de mortalidad para ocho regiones de México 1970. SIC, DGE, México. (Evaluación y análisis III-3.)

Partida, Virgilio (1980), Patrones modelo de mortalidad para México. Tesis de Maestro en Demografía, CEED, El Colegio de México, México.

Reed, Lowell y Merrell, Margaret (1939), "A short method for constructing an adbridged life table" en American Journal of Higiene 30, pp. 33-61.

Zaba, Basia (1979), "The four-parameter logit life table sistem" en Population Studies 33 (1), pp. 79-100. 